\title{
Group elastic symmetries common to continuum and discrete defective crystals
}

\author{
Rachel Nicks · Gareth P Parry
}

Received: date / Accepted: date

\begin{abstract}
The Lie group structure of crystals which have uniform continuous distributions of dislocations allows one to construct associated discrete structures - these are discrete subgroups of the corresponding Lie group, just as the perfect lattices of crystallography are discrete subgroups of $\mathbb{R}^{3}$, with addition as group operation. We consider whether or not the symmetries of these discrete subgroups extend to symmetries of (particular) ambient Lie groups. It turns out that those symmetries which correspond to automorphisms of the discrete structures do extend to (continuous) symmetries of the ambient Lie group (just as the symmetries of a perfect lattice may be embedded in 'homogeneous elastic' deformations). Other types of symmetry must be regarded as 'inelastic'. We show, following Kamber and Tondeur, that the corresponding continuous automorphisms preserve the Cartan torsion, and we characterize the discrete automorphisms by a commutativity condition, (6.14), that relates (via the matrix exponential) to the dislocation density tensor. This shows that periodicity properties of corresponding energy densities are determined by the dislocation density.
\end{abstract}

Keywords Crystals · Defects · Lie Groups

Mathematics Subject Classification (2000) 74A20 - 74E25

Rachel Nicks

School of Mathematics

University of Birmingham, B15 2TT

Tel.: +44-121-4142619

Fax: +44-121-4143389

E-mail: r.nicks@bham.ac.uk

Gareth Parry

School of Mathematical Sciences

University of Nottingham

Nottingham, NG7 2RD

Tel.: +44-115-9513845

Fax: +44-115-9513837

E-mail: gareth.parry@nottingham.ac.uk 


\section{Introduction}

In Davini's continuum model of defective crystals [9], the dislocation density tensor $S=\left(S_{a b}\right), a, b=1,2,3$, is defined by

$$
S_{a b}=\frac{\nabla \wedge \boldsymbol{d}_{a} \cdot \boldsymbol{d}_{b}}{n},
$$

where the fields $\boldsymbol{d}_{1}(\cdot), \boldsymbol{d}_{2}(\cdot), \boldsymbol{d}_{3}(\cdot)$, with $n(\cdot) \equiv \boldsymbol{d}_{1}(\cdot) \cdot \boldsymbol{d}_{2}(\cdot) \wedge \boldsymbol{d}_{3}(\cdot)>0$, are dual to the smooth lattice vector fields $\boldsymbol{\ell}_{1}(\cdot), \boldsymbol{\ell}_{2}(\cdot), \boldsymbol{\ell}_{3}(\cdot)$, which represent the crystal geometry in a region $\Omega$. Each component $S_{a b}$ of the dislocation density tensor is an 'elastic' scalar invariant, in the following sense: if the lattice vector fields $\boldsymbol{\ell}_{a}(\cdot), a=1,2,3$, are transformed via a smooth invertible mapping $\boldsymbol{u}: \Omega \rightarrow$ $\boldsymbol{u}(\Omega) \equiv \Omega^{\prime}$ (the 'elastic deformation') to lattice vector fields $\boldsymbol{\ell}_{a}^{\prime}(\cdot), a=1,2,3$, defined on $\Omega^{\prime}$ by

$$
\boldsymbol{\ell}_{a}^{\prime}(\boldsymbol{u}(\boldsymbol{x}))=\nabla \boldsymbol{u}(\boldsymbol{x}) \boldsymbol{\ell}_{a}(\boldsymbol{x}), \quad \boldsymbol{x} \in \Omega, a=1,2,3,
$$

and if $S_{a b}^{\prime}$ is calculated via the analogue of (1.1), using fields dual to $\ell_{a}^{\prime}(\cdot), a=$ $1,2,3$, then

$$
S_{a b}^{\prime}(\boldsymbol{u}(\boldsymbol{x}))=S_{a b}(\boldsymbol{x}), \quad \boldsymbol{x} \in \Omega, a=1,2,3 .
$$

So, each component of the dislocation density is a scalar invariant - in different language, the value of the dislocation density is unchanged by elastic deformation of the lattice vector fields (defined by (1.2)).

Here, we investigate symmetry issues in a generalization of elasticity theory meant to account for continuous distributions of defects in crystals. We do so by assuming that there is a strain energy density per unit volume in $\Omega$ which is given as a function of arguments denoted $\ell_{1}, \ell_{2}, \ell_{3}, S$, where these arguments are taken to represent values of the fields $\boldsymbol{\ell}_{1}(\cdot), \boldsymbol{\ell}_{2}(\cdot), \boldsymbol{\ell}_{3}(\cdot), S(\cdot)$ at some point in $\Omega$ (the same point for each field). Thus the energy density is expressed in the form

$$
w=w\left(\left\{\ell_{a}\right\}, S\right),
$$

where $\left\{\boldsymbol{\ell}_{a}\right\}$ denotes the set $\left\{\boldsymbol{\ell}_{1}, \boldsymbol{\ell}_{2}, \boldsymbol{\ell}_{3}\right\}$. So in a crystal state $\Sigma$ defined by

$$
\Sigma=\left\{\left\{\boldsymbol{\ell}_{a}(\cdot)\right\} ; \Omega\right\},
$$

where $\left\{\boldsymbol{\ell}_{a}(\cdot)\right\}$ denotes the set of fields $\left\{\boldsymbol{\ell}_{1}(\cdot), \boldsymbol{\ell}_{2}(\cdot), \boldsymbol{\ell}_{3}(\cdot)\right\}$, the energy density at point $\boldsymbol{x}_{0} \in \Omega$ is given by (1.4), where $\boldsymbol{\ell}_{a} \equiv \boldsymbol{\ell}_{a}\left(\boldsymbol{x}_{0}\right), a=1,2,3$, and $S \equiv S\left(\boldsymbol{x}_{0}\right)$.

Note that, in the elastic deformation $\boldsymbol{u}$, from (1.2) and (1.3)

$$
\boldsymbol{\ell}_{a}^{\prime}\left(\boldsymbol{u}\left(\boldsymbol{x}_{0}\right)\right)=\nabla \boldsymbol{u}\left(\boldsymbol{x}_{0}\right) \boldsymbol{\ell}_{a}\left(\boldsymbol{x}_{0}\right), \quad S^{\prime}\left(\boldsymbol{u}\left(\boldsymbol{x}_{0}\right)\right)=S\left(\boldsymbol{x}_{0}\right),
$$

so the energy density at point $\boldsymbol{u}\left(\boldsymbol{x}_{0}\right) \in \Omega^{\prime}$, in the crystal state $\Sigma^{\prime}$ defined by

$$
\Sigma^{\prime}=\left\{\left\{\ell_{a}^{\prime}(\cdot)\right\} ; \Omega^{\prime}\right\},
$$

is

$$
w\left(\left\{\nabla \boldsymbol{u}\left(\boldsymbol{x}_{0}\right) \boldsymbol{\ell}_{a}\right\}, S\right) .
$$


Comparing (1.4) and (1.8), one sees that the dislocation density enters the energy density 'as a parameter' in elastic deformation, in this model of defective crystals, and it is just the deformation gradient, $\nabla \boldsymbol{u}(\cdot)$, that carries information regarding the elastic deformation into the strain energy function. So, one might say that constitutive hypothesis (1.4) represents a 'simple' defective material, c.f. Truesdell and Noll [26], if one accepts that the lattice vector fields transform via (1.2) in an elastic change of crystal state, $\Sigma \rightarrow \Sigma^{\prime}$.

Now if, for some alternative choice of arguments $\left\{\tilde{\ell}_{a}\right\}, \tilde{S}$, say, of the energy density, we have

$$
w\left(\left\{\boldsymbol{\ell}_{a}\right\}, S\right)=w\left(\left\{\tilde{\ell}_{a}\right\}, \tilde{S}\right),
$$

then we say that the mapping $\left(\left\{\boldsymbol{\ell}_{a}\right\}, S\right) \rightarrow\left(\left\{\tilde{\ell_{a}}\right\}, \tilde{S}\right)$ is a symmetry of $w$. Throughout this paper, we shall take the view that the symmetries of the continuum energy density $w$ are determined by the geometrical symmetries of a discrete structure which is associated with a particular crystal state determined by the arguments of $w$. This is a generalization of the commonly held idea that a continuum energy density that models a perfect crystal $(S=0)$ has symmetries determined by the geometrical symmetries of a perfect lattice associated with a crystal state determined by the arguments $\left(\left\{\boldsymbol{\ell}_{a}\right\}, 0\right)$ of the energy density. The perfect lattice is taken to have (integral) basis $\boldsymbol{\ell}_{1}, \boldsymbol{\ell}_{2}, \boldsymbol{\ell}_{3}$ and the geometrical symmetries of the lattice correspond to the different choices of integral basis. (Note that the changes of basis extend to linear transformations of $\mathbb{R}^{3}$ to itself). In this particular case, then, we have that

$$
w\left(\left\{\boldsymbol{\ell}_{a}\right\}, 0\right)=w\left(\left\{\boldsymbol{\ell}_{a}^{\prime}\right\}, 0\right)
$$

provided that there exists a matrix $\gamma=\left(\gamma_{a b}\right) \in G L_{3}(\mathbb{Z})$ (i.e. $\gamma$ has integer entries and determinant \pm 1 ) such that

$$
\ell_{a}^{\prime}=\gamma_{a b} \ell_{b}, \quad a=1,2,3
$$

where repeated indices run from 1 to 3 in (1.11) and throughout.

Notice that there is a choice involved, when on 'associates' a perfect lattice with a crystal state determined by the arguments $\left(\left\{\boldsymbol{\ell}_{a}\right\}, 0\right)$. Indeed, a crystal state ( $\Sigma^{c}$ say) where the corresponding lattice vector fields $\ell_{a}^{c}(\cdot)$ are constant, $\boldsymbol{\ell}_{a}^{c}(\boldsymbol{x})=\boldsymbol{\ell}_{a}, a=1,2,3, \boldsymbol{x} \in \Omega$, has $S=0$, but by the elastic invariance of $S(\cdot)$, all crystal states elastically related to $\Sigma^{c}$ also have vanishing dislocation density. The perfect lattice with integral basis $\ell_{1}, \ell_{2}, \ell_{3}$ has a particular status with respect to $\Sigma^{c}$ : the constant fields $\ell^{c}(\cdot)$ are translation invariant $\left(\ell_{a}^{c}(\boldsymbol{x})=\ell_{a}^{c}(\boldsymbol{x}+\boldsymbol{y}), \boldsymbol{x}, \boldsymbol{y} \in \mathbb{R}^{3}\right)$ or, said differently, they are invariant in the sense that

$$
\ell_{a}^{c}(\boldsymbol{\psi}(\boldsymbol{x}, \boldsymbol{y}))=\nabla_{1} \boldsymbol{\psi}(\boldsymbol{x}, \boldsymbol{y}) \ell_{a}^{c}(\boldsymbol{x}), \quad \boldsymbol{\psi}(\boldsymbol{x}, \boldsymbol{y}) \equiv \boldsymbol{x}+\boldsymbol{y} .
$$

(In (1.12), $\nabla_{1}$ represents the gradient with respect to the first argument). Relation (1.12) expresses the right invariance of the fields $\ell_{a}^{c}(\cdot)$ in the Lie group that is $\mathbb{R}^{3}$ with addition as group operation (we denote it $\left(\mathbb{R}^{3},+\right)$ ) and the perfect lattices are the (only) discrete subgroups of $\left(\mathbb{R}^{3},+\right)$. From this 
perspective, associating a perfect lattice with arguments $\left(\left\{\boldsymbol{\ell}_{a}\right\}, 0\right)$ involves choosing a particular canonical crystal state $\Sigma^{c}$ from amongst those crystal states which have zero dislocation density, noting that the fields $\ell_{a}^{c}(\cdot)$ are right invariant fields in a certain Lie group, and finding the corresponding discrete subgroups.

This construction may be generalized to the case $S \neq 0$ as follows. Assume that the arguments $\left(\left\{\boldsymbol{\ell}_{a}\right\}, S\right)$ of $w$ correspond to values of fields $\boldsymbol{\ell}_{a}(\cdot), S(\cdot)$ at a particular point, $\mathbf{0}$ say, with $S(\cdot)$ calculated from $\ell_{a}(\cdot)$ via the dual vector fields $\boldsymbol{d}_{a}(\cdot)$ and (1.1). Furthermore, assume that the lattice vector fields are such that $S(\cdot)$ is constant in the relevant region, since no information regarding the derivatives of $S(\cdot)$ is given as an argument of $w$ (if the constitutive assumption (1.4) were different, this further assumption would not be appropriate). We shall say that crystal states where $S(\cdot)$ is constant are uniform crystal states. It follows, according to Pontryagin [25], that the partial differential system

$$
\boldsymbol{\ell}_{a}(\boldsymbol{\psi}(\boldsymbol{x}, \boldsymbol{y}))=\nabla_{1} \boldsymbol{\psi}(\boldsymbol{x}, \boldsymbol{y}) \boldsymbol{\ell}_{a}(\boldsymbol{x}), \boldsymbol{x}, \boldsymbol{y} \in \Omega, \quad a=1,2,3,
$$

has a unique solution $\psi: \Omega \rightarrow \Omega$ which has the properties of Lie group composition function with $\mathbf{0}$ acting as group identity. In (1.13) the fields $\left\{\boldsymbol{\ell}_{a}(\cdot)\right\}$ are fields given in some uniform crystal state. Thus

$$
\boldsymbol{\psi}(\boldsymbol{\psi}(\boldsymbol{x}, \boldsymbol{y}), \boldsymbol{z})=\boldsymbol{\psi}(\boldsymbol{x}, \boldsymbol{\psi}(\boldsymbol{y}, \boldsymbol{z})), \boldsymbol{\psi}(\boldsymbol{x}, \mathbf{0})=\boldsymbol{\psi}(\mathbf{0}, \boldsymbol{x})=\boldsymbol{x}, \boldsymbol{x}, \boldsymbol{y}, \boldsymbol{z} \in \Omega
$$

and there exists an element $\boldsymbol{x}^{-1} \in \Omega$ such that

$$
\boldsymbol{\psi}\left(\boldsymbol{x}, \boldsymbol{x}^{-1}\right)=\boldsymbol{\psi}\left(\boldsymbol{x}^{-1}, \boldsymbol{x}\right)=\mathbf{0} .
$$

We shall often find it more convenient to write, below, that

$$
\psi(x, y) \equiv x y,
$$

so that juxtaposition represents group multiplication. Then we have from (1.14) and (1.15) that

$$
(\boldsymbol{x y}) \boldsymbol{z}=\boldsymbol{x}(\boldsymbol{y} z), \boldsymbol{x} 0=\mathbf{0 x}=\boldsymbol{x}, \boldsymbol{x} \boldsymbol{x}^{-1}=\boldsymbol{x}^{-1} \boldsymbol{x}=\mathbf{0} .
$$

We shall confine attention, here, exclusively to the case where $\Omega \equiv \mathbb{R}^{3}$ and denote the corresponding Lie group by $G \equiv\left(\mathbb{R}^{3}, \boldsymbol{\psi}\right)$.

Again, by the elastic invariance of the dislocation density, there is an infinite choice of lattice vector fields which have given, constant, $S$ - all (uniform) crystal states elastically related to a given uniform crystal state have the same (constant) dislocation density. For each such choice of lattice vector fields, there is a corresponding Lie group structure, obtained by solution of (1.13). In the general case, we shall make a 'canonical' choice from amongst those Lie groups obtained, via (1.13), from lattice vector fields elastically related to each other via (1.2) (for given $S$ ). We then find the discrete subgroups of this canonical group - so these discrete subgroups provide the analogues of the perfect lattices of traditional crystallography. 
To describe the class of elastically related uniform crystal states in a little more detail, suppose that $\left\{\ell_{a}^{\prime}(\cdot)\right\}$ and $\left\{\boldsymbol{\ell}_{a}(\cdot)\right\}$ are related via (1.2). Then, cf. $(1.13)$

$$
\ell_{a}^{\prime}\left(\boldsymbol{\psi}^{\prime}(\boldsymbol{r}, \boldsymbol{s})\right)=\nabla_{1} \boldsymbol{\psi}^{\prime}(\boldsymbol{r}, \boldsymbol{s}) \boldsymbol{\ell}_{a}(\boldsymbol{r}), \boldsymbol{r}, \boldsymbol{s} \in \mathbb{R}^{3}, \quad a=1,2,3,
$$

if $\boldsymbol{\psi}^{\prime}: \mathbb{R}^{3} \times \mathbb{R}^{3} \rightarrow \mathbb{R}^{3}$ is defined by

$$
\psi^{\prime}(\boldsymbol{r}, \boldsymbol{s})=\boldsymbol{u}\left(\boldsymbol{\psi}\left(\boldsymbol{u}^{-1}(\boldsymbol{r}), \boldsymbol{u}^{-1}(\boldsymbol{s})\right)\right) .
$$

Relations (1.13), (1.19) express the right invariance of the fields $\left\{\boldsymbol{\ell}_{a}(\cdot)\right\},\left\{\boldsymbol{\ell}_{a}^{\prime}(\cdot)\right\}$ with respect to the Lie groups $G \equiv\left(\mathbb{R}^{3}, \boldsymbol{\psi}\right), G^{\prime} \equiv\left(\mathbb{R}^{3}, \boldsymbol{\psi}^{\prime}\right)$ respectively. Since the mapping $\boldsymbol{u}: \mathbb{R}^{3} \rightarrow \mathbb{R}^{3}$ is invertible, the groups $G$ and $G^{\prime}$ are isomorphic, i.e. (1.19) holds. Choosing one uniform crystal state amongst those elastically related to it therefore amounts to choosing one Lie group amongst those which are isomorphic to it.

Recall that, given a group $G$ with composition function $\boldsymbol{\psi}$, the structure constants $C_{i j k}$ of the corresponding Lie algebra $\mathfrak{g}$ are given by

$$
C_{i j k}=\frac{\partial^{2} \psi_{i}}{\partial x_{j} \partial y_{k}}(\mathbf{0}, \mathbf{0})-\frac{\partial^{2} \psi_{i}}{\partial x_{k} \partial y_{j}}(\mathbf{0}, \mathbf{0})
$$

where $\boldsymbol{\psi}=\psi_{i}(\boldsymbol{x}, \boldsymbol{y}) \boldsymbol{e}_{i}$, and the Lie bracket operation $[\cdot, \cdot]: \mathbb{R}^{3} \times \mathbb{R}^{3} \rightarrow \mathbb{R}^{3}$ is defined by

$$
[\boldsymbol{x}, \boldsymbol{y}]=C_{i j k} x_{j} y_{k} \boldsymbol{e}_{i}, \quad \boldsymbol{x}, \boldsymbol{y} \in \mathbb{R}^{3},
$$

with respect to some basis $\left\{\boldsymbol{e}_{1}, \boldsymbol{e}_{2}, \boldsymbol{e}_{3}\right\}$ of $\mathbb{R}^{3}$. The vector space $\mathbb{R}^{3}$ and the operation $[\cdot, \cdot]$ make up the Lie algebra $\mathfrak{g}$ corresponding to the Lie group $G=$ $\left(\mathbb{R}^{3}, \boldsymbol{\psi}\right)$.

The connection between the dislocation density tensor $S$, defined via (1.1), and the structure constants is

$$
C_{i j k} \ell_{r j}(\mathbf{0}) \ell_{s k}(\mathbf{0})=\epsilon_{p r s} S_{k p} \ell_{k i}(\mathbf{0}),
$$

where $\epsilon_{p r s}$ is the permutation symbol and $\boldsymbol{\ell}_{r}(\mathbf{0})=\ell_{r j}(\mathbf{0}) \boldsymbol{e}_{j}$, see Elzanowski and Parry [10].

Let $\mathfrak{g}$ and $\mathfrak{g}^{\prime}$ be Lie algebras with Lie brackets $[\cdot, \cdot]_{\mathfrak{g}},[\cdot, \cdot]_{\mathfrak{g}^{\prime}}$ respectively. A Lie algebra isomorphism is an invertible linear transformation $L: \mathfrak{g} \rightarrow \mathfrak{g}^{\prime}$ which satisfies

$$
[L \boldsymbol{x}, L \boldsymbol{y}]_{\mathfrak{g}^{\prime}}=L[\boldsymbol{x}, \boldsymbol{y}]_{\mathfrak{g}}, \quad \boldsymbol{x}, \boldsymbol{y} \in \mathfrak{g}
$$

If $C_{i j k}^{\mathfrak{g}}, C_{i j k}^{\mathfrak{g}^{\prime}}$ are the structure constants for $\mathfrak{g}, \mathfrak{g}^{\prime}$ respectively, then (1.21) implies that

$$
C_{i j k}^{\mathfrak{g}^{\prime}} L_{j p} L_{k q}=L_{i r} C_{r p q}^{\mathfrak{g}},
$$

where $L \boldsymbol{e}_{i}=L_{j i} \boldsymbol{e}_{j}, i=1,2,3$. If $\mathfrak{g}^{\prime}$ is the Lie algebra of $G^{\prime}=\left(\mathbb{R}^{3}, \boldsymbol{\psi}^{\prime}\right)$, and $\boldsymbol{u}: \mathbb{R}^{3} \rightarrow \mathbb{R}^{3}$ is a Lie group isomorphism, then $\nabla \boldsymbol{u}(\mathbf{0}) \equiv L$ is a Lie algebra isomorphism. Conversely, if an invertible linear transformation $L$ satisfies (1.23), then there exists a Lie group isomorphism $\boldsymbol{u}$ such that $\nabla \boldsymbol{u}(\mathbf{0})=L$. 
Now, in the case that $S=0$, it is clear that the symmetries of the perfect lattices are (restrictions) of elastic deformations - for any change of basis $\boldsymbol{\ell}_{a} \rightarrow \gamma_{a b} \boldsymbol{\ell}_{b} \equiv \boldsymbol{\ell}_{a}^{\prime}, \gamma=\left(\gamma_{a b}\right) \in G L_{3}(\mathbb{Z})$, extends to a homogeneous deformation $F$ of $\mathbb{R}^{3}$ to itself defined by

$$
F\left(x_{a} \ell_{a}\right)=x_{a}\left(F \ell_{a}\right) \equiv x_{a} \ell_{a}^{\prime},
$$

$x_{a} \in \mathbb{R}, a=1,2,3$

We shall investigate whether or not it is true, in the case $S \neq 0$, that symmetries of the energy density (which we interpret as geometrical symmetries of the discrete subgroups of $\left.\left(\mathbb{R}^{3}, \boldsymbol{\psi}\right)\right)$ extend to elastic deformations of $\mathbb{R}^{3}$ to itself, loosely whether or not particular symmetries are elastic or 'inelastic'. This question is important, it seems to us, because it highlights inelastic processes which preserve the discrete structure of the crystal, and such processes (e.g. slip in directions determined by the lattice geometry and the Burger's vectors) are essential building blocks in phenomenological, thermomechanical, theories of crystal behaviour.

To simplify matters a little, we restrict attention to the canonical group in the isomorphism class of Lie groups defined by $S$, and consider whether or not the symmetries of the discrete subgroup extend to automorphisms of the canonical group. In fact we concentrate on a particular class of (solvable) Lie groups, cf. Nicks and Parry [19], and show that some symmetries of the discrete subgroup can indeed be extended to automorphisms of $G$, and that others cannot be so extended i.e some symmetries of the discrete structure are elastic, some are inelastic.

We begin the work by recalling, in section 2 , how to construct discrete subgroups of a Lie group, and providing definitions of solvable Lie groups and algebras. Next, we recall the definition of Cartan torsion, in this context, and show that the mappings that preserve the Cartan torsion include the group automorphisms, cf. Kamber and Tondeur [14]. We introduce the solvable group $S_{1}$, following Auslander et al [1], who showed that there are only three classes of Lie group which have discrete subgroups (in three dimensions). We calculate the automorphisms of $S_{1}$ and its Lie algebra $\mathfrak{s}_{1}$ and factorise the automorphisms of $S_{1}$ (uniquely) as compositions of (matrix exponentials of) certain rotations, 'shears' and 'pure strains'. These calculations are necessary prerequisites for discussion of the symmetries of canonical discrete subgroups $D$ of $S_{1}$.

We show in section 5 that a discrete subgroups $D$ of $S_{1}$ can be identified with points of a perfect lattice $\mathbb{Z}^{3}$, even though the corresponding continuum crystal state has $S \neq 0$. In this context, of course, the lattice vector fields are not commutative, and the Burgers' vectors $\nabla \wedge \boldsymbol{d}_{a}$ are nonzero, as are the Lie brackets of (pairs of) the lattice vector fields. The discrete analogues of the Lie brackets are the commutators of elements of the discrete group $D$, and we note that the commutator relations (5.2) below (which define $D$ ) involve quantities $a, b, c, d$ which are related to the dislocation density via (4.4), (4.18). The commutator subgroup, denoted $D^{\prime}$, of $D$ is in this case an abelian group generated by the commutators of the generators of $D$-it can be identified with 
points of $\mathbb{Z}^{2}$ in this case. The elements of $D^{\prime}$ represent points which may be reached by composition of 'discrete Burgers circuits' in the defective crystal. It will become apparent that the arguments $\left(\left\{\boldsymbol{\ell}_{a}\right\}, S\right)$ of the energy density represent quantities that relate to the Lie algebra $\mathfrak{s}_{1}$, also that exponentiation of the algebra elements $\left\{\boldsymbol{\ell}_{a}\right\}$ gives generators of the discrete subgroup $D$. As noted above, the dislocation density gives us the (infinitesimal version of the) commutator relations, and thus the commutator subgroup $D^{\prime}$.

Next, we consider the symmetries of the discrete subgroup $D$. Given that we restrict attention to a particular canonical Lie group $G=\left(\mathbb{R}^{3}, \boldsymbol{\psi}\right)$, and given the above interpretation of the arguments of $w$, the task in fact requires that we find all sets of generators of $D$. We give an algorithm which, given any set of generators, puts the set in the form (5.5), and quote necessary and sufficient conditions, from Nicks and Parry [19], that group elements of the form (5.5) generate $D$. Then, any set of generators is obtained from a set of the form (5.5) (satisfying the necessary and sufficient conditions given in [19]) by a sequence of elementary operations - the Nielsen transformations described in Johnson [13].

In section 6 , we improve upon a result given in [19] by invoking the 'substitution test' of Johnson [13] to give necessary and sufficient conditions that any symmetry of $D$ (i.e. any change of generators of $D$ ) extend to an automorphism of $S_{1}$. Finally we use these conditions to identify those changes of generators that do extend to automorphisms of $S_{1}$, and those that do not. This involves finding the matrices $M \in G L_{2}(\mathbb{Z})$ which commute with a given matrix $\theta \in G L_{2}(\mathbb{Z})$ related to the dislocation density - this is a number theoretic problem studied by Baake and Roberts [2], so we summarize results of theirs which are relevant to this work.

\section{Construction of discrete subgroups}

Vector fields $\boldsymbol{\nu}(\cdot)$ which satisfy

$$
\boldsymbol{\nu}(\boldsymbol{\psi}(\boldsymbol{x}, \boldsymbol{y}))=\nabla_{1} \boldsymbol{\psi}(\boldsymbol{x}, \boldsymbol{y}) \boldsymbol{\nu}(\boldsymbol{x}), \quad \boldsymbol{x} \in \mathbb{R}^{3}
$$

are said to be right invariant on $G$. (Recall that we take $\Omega \equiv \mathbb{R}^{3}$ throughout). From (1.13) one sees that the lattice vector fields $\ell_{1}(\cdot), \ell_{2}(\cdot), \ell_{3}(\cdot)$ are right invariant. In fact these fields provide a basis for the vector space of all right invariant vector fields on $G$. Let $\boldsymbol{\nu}(\boldsymbol{x})=\nu_{a} \boldsymbol{\ell}_{a}(\boldsymbol{x})$ for real numbers $\nu_{a} a=1,2,3$ and define the integral curve through $\boldsymbol{x}_{0}$ of the field $\boldsymbol{\nu}(\cdot)$ to be the solution $\{\boldsymbol{x}(t): t \in \mathbb{R}\}$ of the ordinary differential equation $\dot{\boldsymbol{x}}(t)=\nu_{a} \boldsymbol{\ell}_{a}(\boldsymbol{x}(t)), \boldsymbol{x}(0)=$ $\boldsymbol{x}_{0}$. Note that $\boldsymbol{\nu}:=\boldsymbol{\nu}(\mathbf{0})$ determines the field $\boldsymbol{\nu}(\boldsymbol{x})$ by (2.1). One can then define the mapping $\exp (\boldsymbol{\nu}): G \rightarrow G$ by

$$
\exp (\boldsymbol{\nu})\left(\boldsymbol{x}_{0}\right)=\boldsymbol{x}(1),
$$

and the group element $\mathrm{e}^{(\boldsymbol{\nu})}$ by

$$
\mathrm{e}^{(\boldsymbol{\nu})}=\exp (\boldsymbol{\nu})(\mathbf{0}) .
$$


Also, note that $\mathrm{e}^{(\cdot)}: \mathfrak{g} \rightarrow G$ is called the exponential mapping of the Lie algebra to the Lie group. It is standard result of Lie group theory that

$$
\exp (\boldsymbol{\nu})(\boldsymbol{x})=\boldsymbol{\psi}\left(\mathrm{e}^{(\boldsymbol{\nu})}, \boldsymbol{x}\right)
$$

and this states that the flow along the integral curves of the lattice vector fields corresponds to group multiplication by the group element $\mathrm{e}^{(\boldsymbol{\nu})}$. In the case of perfect crystals, choosing $\boldsymbol{\ell}_{a}(\cdot) \equiv \boldsymbol{\ell}_{a}(\mathbf{0}) \equiv \boldsymbol{e}_{a}$ for a basis $\left\{\boldsymbol{e}_{1}, \boldsymbol{e}_{2}, \boldsymbol{e}_{3}\right\}$ of $\mathbb{R}^{3}$, iterating the flow along the lattice vector fields (which in this case is just translation by $\left.\boldsymbol{e}_{1}, \boldsymbol{e}_{2}, \boldsymbol{e}_{3}\right)$ produces a perfect lattice. In the case of $G=\left(\mathbb{R}^{3}, \boldsymbol{\psi}\right)$ the analogue of the perfect lattice is the set of points (or group elements) produced by iterating the flow (from $t=0$ to $t=1$ ) along the lattice vector fields, starting at the origin. By (2.2)-(2.4) one obtains the subgroup of $G$ that is generated by the group elements $\mathrm{e}^{\left(\ell_{1}\right)}, \mathrm{e}^{\left(\ell_{2}\right)}, \mathrm{e}^{\left(\ell_{3}\right)}$ where $\boldsymbol{\ell}_{a}=\boldsymbol{\ell}_{a}(\mathbf{0})$, $a=1,2,3$.

If $\mathfrak{g}$ is the Lie algebra of $G$ and $\mathfrak{g}^{\prime}$ is the Lie algebra of $G^{\prime}$ and $\phi: G \rightarrow G^{\prime}$ is a Lie group homomorphism, $\nabla \boldsymbol{\phi}(\mathbf{0}) \equiv L$ is a Lie algebra homomorphism. Conversely if $L$ satisfies (1.23) then there exists a Lie group homomorphism $\phi$ such that $\nabla \phi(\mathbf{0})=L$. In addition

$$
\phi\left(\mathrm{e}^{(\boldsymbol{\nu})}\right)=\mathrm{e}^{(\nabla \phi(0) \boldsymbol{\nu})}, \quad \boldsymbol{\nu} \in \mathfrak{g},
$$

where the exponential on the left hand side is the exponential which maps $\mathfrak{g}$ to $G$ and that on the right hand side maps $\mathfrak{g}^{\prime}$ to $G^{\prime}$.

\section{Invariance of torsion $\mathbb{T}$}

The Cartan torsion is the tensor $\mathbb{T}$ defined, following Davini [8], by

$$
\mathbb{T}(\cdot)=\left(\nabla \wedge \boldsymbol{d}_{a}(\cdot)\right) \otimes \boldsymbol{\ell}_{a}(\cdot)
$$

It is a measure of the non-commutativity of the lattice vector fields which is related to the dislocation density $S$ and the Burgers' vectors $\nabla \wedge \boldsymbol{d}_{a}$ by (3.1). Also

$$
\nabla \wedge \boldsymbol{d}_{a}(\cdot)=\mathbb{T}(\cdot) \boldsymbol{d}_{a}(\cdot)=n(\cdot) S_{a b}(\cdot) \boldsymbol{\ell}_{b}(\cdot)
$$

If we define the quantities

$$
\mathcal{L}_{a}=\epsilon_{a b c}\left(\ell_{c} \cdot \nabla\right) \ell_{b},
$$

(noting that $\mathcal{L}_{1}$ is the Lie bracket of the fields $\ell_{3}$ and $\ell_{2}$, etc.), then we have that

$$
\mathbb{T}(\cdot)=\left(\nabla \wedge \boldsymbol{d}_{a}(\cdot)\right) \otimes \boldsymbol{\ell}_{a}(\cdot)=\boldsymbol{n} \boldsymbol{\ell}_{a} \otimes \mathcal{L}_{a} .
$$

(This definition of torsion incorporates a factor of $\frac{1}{2}$ which does not appear in some other sources.) Let $\boldsymbol{u}: \mathbb{R}^{3} \rightarrow \mathbb{R}^{3}$ be an elastic deformation and note that 
vector fields $\boldsymbol{\ell}_{a}$ deform as in (1.2) and other associated quantities behave as follows:

$$
\begin{aligned}
\left(\frac{\nabla \wedge \boldsymbol{d}_{a}}{\boldsymbol{n}}\right)^{\prime}(\boldsymbol{u}(\boldsymbol{x})) & =\nabla \boldsymbol{u}(\boldsymbol{x})\left(\frac{\nabla \wedge \boldsymbol{d}_{a}}{\boldsymbol{n}}\right)(\boldsymbol{x}), \\
\boldsymbol{n}^{\prime}(\boldsymbol{u}(\boldsymbol{x})) & =(\operatorname{det} \nabla \boldsymbol{u}(\boldsymbol{x}))^{-1} \boldsymbol{n}(\boldsymbol{x}), \\
\mathcal{L}_{a}^{\prime}(\boldsymbol{u}(\boldsymbol{x})) & =\nabla \boldsymbol{u}(\boldsymbol{x}) \mathcal{L}_{a}(\boldsymbol{x}) .
\end{aligned}
$$

If we interpret elastic deformation as coordinate transformation, we may say that $\mathbb{T}$ behaves as does a relative tensor of type $(2,0)$ and weight -1 in Lovelock and Rund's terminology [16]. Investigating the invariance properties of $\mathbb{T}$ then amounts to solving

$$
\mathbb{T}(\boldsymbol{\tau}(\boldsymbol{x}))=\nabla \boldsymbol{\tau}(\boldsymbol{x}) \mathbb{T}(\boldsymbol{x})(\nabla \boldsymbol{\tau}(\boldsymbol{x}))^{T}(\operatorname{det} \nabla \boldsymbol{\tau}(\boldsymbol{x}))^{-1}
$$

for smooth invertible functions $\tau: \mathbb{R}^{3} \rightarrow \mathbb{R}^{3}$. Condition (3.8) is the requirement that the elastic deformation $\boldsymbol{\tau}$ leaves the field $\mathbb{T}$ unchanged (i.e. $\left.\mathbb{T}^{\prime}(\boldsymbol{\tau}(\boldsymbol{x}))=\mathbb{T}(\boldsymbol{\tau}(\boldsymbol{x}))\right)$. We proceed in stages, adapting Kamber and Tondeur a little [14].

i) By hypothesis the fields $\ell_{a}(\cdot)$ are right invariant so that (1.13) is satisfied and the analogues of (3.5)-(3.7) also hold. Thus, for an arbitrary fixed $\boldsymbol{y} \in \mathbb{R}^{3}, \boldsymbol{\tau}(\boldsymbol{x})=\boldsymbol{\psi}(\boldsymbol{x}, \boldsymbol{y})$ solves (3.8). Moreover, if $\boldsymbol{\tau}$ is any given solution of (3.8) then $\boldsymbol{\tau}^{*}$ defined by $\boldsymbol{\tau}^{*}(\boldsymbol{x})=\boldsymbol{\psi}(\boldsymbol{\tau}(\boldsymbol{x}), \boldsymbol{y}$ ) also solves (3.8):

$$
\begin{aligned}
\mathbb{T}\left(\boldsymbol{\tau}^{*}(\boldsymbol{x})\right) & =\mathbb{T}(\boldsymbol{\psi}(\boldsymbol{\tau}(\boldsymbol{x}), \boldsymbol{y})) \\
& =\nabla_{1} \boldsymbol{\psi}(\boldsymbol{\tau}(\boldsymbol{x}), \boldsymbol{y}) \mathbb{T}(\boldsymbol{\tau}(\boldsymbol{x}))\left(\nabla_{1} \boldsymbol{\psi}(\boldsymbol{\tau}(\boldsymbol{x}), \boldsymbol{y})\right)^{T}\left(\operatorname{det}\left(\nabla_{1} \boldsymbol{\psi}(\boldsymbol{\tau}(\boldsymbol{x}), \boldsymbol{y})\right)^{-1}\right. \\
& =\nabla_{1} \boldsymbol{\psi}(\boldsymbol{\tau}(\boldsymbol{x}), \boldsymbol{y}) \nabla \boldsymbol{\tau}(\boldsymbol{x}) \mathbb{T}(\boldsymbol{x})(\nabla \boldsymbol{\tau}(\boldsymbol{x}))^{T}\left(\nabla_{1} \boldsymbol{\psi}(\boldsymbol{\tau}(\boldsymbol{x}), \boldsymbol{y})\right)^{T} \\
& \quad\left(\operatorname{det}\left(\nabla_{1} \boldsymbol{\psi}(\boldsymbol{\tau}(\boldsymbol{x}), \boldsymbol{y})\right)^{-1}(\operatorname{det} \nabla \boldsymbol{\tau}(\boldsymbol{x}))^{-1}\right. \\
& =\nabla \boldsymbol{\tau}^{*}(\boldsymbol{x}) \mathbb{T}(\boldsymbol{x})\left(\nabla \boldsymbol{\tau}^{*}(\boldsymbol{x})\right)^{T}\left(\operatorname{det} \nabla \boldsymbol{\tau}^{*}(\boldsymbol{x})\right)^{-1} .
\end{aligned}
$$

In the multiplicative notation where $\boldsymbol{\psi}(\boldsymbol{x}, \boldsymbol{y}) \equiv \boldsymbol{x} \boldsymbol{y}$ we have $\boldsymbol{\tau}^{*}=\boldsymbol{\tau} \boldsymbol{y}$ so $\boldsymbol{\tau}^{*}(\mathbf{0})=\boldsymbol{\tau}(\mathbf{0}) \boldsymbol{y}$. Choose $\boldsymbol{y}=\left(\boldsymbol{\tau}^{*}(\mathbf{0})\right)^{-1}$ so that $\boldsymbol{\tau}^{*}(\mathbf{0})=\mathbf{0}$ and then

$$
\boldsymbol{\tau}=\boldsymbol{\tau}^{*} \boldsymbol{y}^{-1}=\boldsymbol{\psi}\left(\boldsymbol{\tau}^{*}, \boldsymbol{\tau}(\mathbf{0})\right) .
$$

According to (3.9) any solution $\boldsymbol{\tau}$ of (3.8) is expressible as the product of a solution $\boldsymbol{\tau}^{*}$ of (3.8) which has the property $\boldsymbol{\tau}^{*}(\mathbf{0})=\mathbf{0}$ with a group element $\boldsymbol{\tau}(\mathbf{0})$. So it suffices, in order to obtain the general solution of (3.8) just to find those solutions $\boldsymbol{\tau}$ of (3.8) which are such that $\boldsymbol{\tau}(\mathbf{0})=\mathbf{0}$.

ii) From (3.8), putting $\boldsymbol{x}=\mathbf{0}$ and noting that $\boldsymbol{\tau}(\mathbf{0})=\mathbf{0}$ we get

$$
\mathbb{T}(\mathbf{0})=\nabla \boldsymbol{\tau}(\mathbf{0}) \mathbb{T}(\mathbf{0})(\nabla \boldsymbol{\tau}(\mathbf{0}))^{T}(\operatorname{det} \nabla \boldsymbol{\tau}(\mathbf{0}))^{-1} .
$$

We can also show from the definition (3.1) that $\mathbb{T}_{i j}(\mathbf{0})=\frac{1}{2} \epsilon_{i r s} C_{j r s}$ where $C_{j r s}$ are the structure constants of the Lie algebra. We therefore find that

$$
(\nabla \boldsymbol{\tau}(\mathbf{0}))_{i r} C_{r p q}=C_{i j k}(\nabla \boldsymbol{\tau}(\mathbf{0}))_{j p}(\nabla \boldsymbol{\tau}(\mathbf{0}))_{k q}
$$

so that by (1.24) $L=\nabla \boldsymbol{\tau}(\mathbf{0})$ is a Lie algebra automorphism since we confine attention to smooth invertible $\boldsymbol{\tau}$ with $\boldsymbol{\tau}(\mathbf{0})=\mathbf{0}$. 
iii) Finally we show that all Lie group automorphisms solve (3.8). Define $R(\boldsymbol{x})=\nabla_{1} \boldsymbol{\psi}(\mathbf{0}, \boldsymbol{x})$ and note that since $\boldsymbol{\psi}(\boldsymbol{x}, \boldsymbol{y})$ solves (3.8) for arbitrary fixed $\boldsymbol{y}$, putting $\boldsymbol{x}=\mathbf{0}$ in (3.8) with $\boldsymbol{\tau}(\boldsymbol{x})=\boldsymbol{\psi}(\boldsymbol{x}, \boldsymbol{y})$ gives $\boldsymbol{\tau}(\mathbf{0})=\boldsymbol{\psi}(\mathbf{0}, \boldsymbol{y})=$ $\boldsymbol{y}$ and

$$
\mathbb{T}(\boldsymbol{y})=R(\boldsymbol{y}) \mathbb{T}(\mathbf{0}) R(\boldsymbol{y})^{T}(\operatorname{det} R(\boldsymbol{y}))^{-1} .
$$

Now let $\boldsymbol{\tau}$ be any Lie group automorphism so that

$$
\boldsymbol{\tau}(\boldsymbol{\psi}(\boldsymbol{x}, \boldsymbol{y}))=\boldsymbol{\psi}(\boldsymbol{\tau}(\boldsymbol{x}), \boldsymbol{\tau}(\boldsymbol{y}))
$$

and $\boldsymbol{\tau}(\mathbf{0})=(\mathbf{0})$. Differentiating (3.12) with respect to $\boldsymbol{x}$ and putting $\boldsymbol{x}=\mathbf{0}$ gives

$$
\nabla \boldsymbol{\tau}(\boldsymbol{y}) R(\boldsymbol{y})=R(\boldsymbol{\tau}(\boldsymbol{y})) \nabla \boldsymbol{\tau}(\mathbf{0}) .
$$

From (3.11)-(3.13) we then find that

$$
\begin{aligned}
\mathbb{T}(\boldsymbol{\tau}(\boldsymbol{y}))= & R(\boldsymbol{\tau}(\boldsymbol{y})) \mathbb{T}(\mathbf{0}) R(\boldsymbol{\tau}(\boldsymbol{y}))^{T}(\operatorname{det} R(\boldsymbol{\tau}(\boldsymbol{y})))^{-1} \\
= & \nabla \boldsymbol{\tau}(\boldsymbol{y}) R(\boldsymbol{y})(\nabla \boldsymbol{\tau}(\mathbf{0}))^{-1} \mathbb{T}(\mathbf{0})(\nabla \boldsymbol{\tau}(\mathbf{0}))^{-T} R(\boldsymbol{y})^{T}(\nabla \boldsymbol{\tau}(\boldsymbol{y}))^{T} \\
& \quad(\operatorname{det} \nabla \boldsymbol{\tau}(\boldsymbol{y}))^{-1}(\operatorname{det} R(\boldsymbol{y}))^{-1}(\operatorname{det} \nabla \boldsymbol{\tau}(\mathbf{0})) \\
= & \nabla \boldsymbol{\tau}(\boldsymbol{y}) R(\boldsymbol{y}) \mathbb{T}(\mathbf{0}) R(\boldsymbol{y})^{T}(\nabla \boldsymbol{\tau}(\boldsymbol{y}))^{T}(\operatorname{det} \nabla \boldsymbol{\tau}(\boldsymbol{y}))^{-1}(\operatorname{det} R(\boldsymbol{y}))^{-1} \\
= & \nabla \boldsymbol{\tau}(\boldsymbol{y}) \mathbb{T}(\boldsymbol{y})(\nabla \boldsymbol{\tau}(\boldsymbol{y}))^{T}(\operatorname{det} \nabla \boldsymbol{\tau}(\boldsymbol{y}))^{-1}
\end{aligned}
$$

so that (3.8) holds for such choices of $\boldsymbol{\tau}$.

Thus the smooth invertible solutions of (3.8) that preserve the Cartan Torsion are the functions obtained by right multiplication of the group automorphisms.

\section{Solvable Lie groups}

We shall be concerned with the the automorphisms of a class of solvable Lie groups, so here we give relevant definitions.

If $\mathfrak{g}$ is a Lie algebra, and one defines $\mathfrak{g}_{1}=\mathfrak{g}, \mathfrak{g}_{2}=\left[\mathfrak{g}_{1}, \mathfrak{g}_{1}\right], \ldots, \mathfrak{g}_{k}=$ $\left[\mathfrak{g}_{k-1}, \mathfrak{g}_{k-1}\right]$, then $\mathfrak{g}$ is called solvable if $\mathfrak{g}_{k}=\mathbf{0}$ for some $k$. Similarly, let $G$ be a connected Lie group and let $(\boldsymbol{x}, \boldsymbol{y})=\boldsymbol{x}^{-1} \boldsymbol{y}^{-1} \boldsymbol{x} \boldsymbol{y}$ denote the commutator of $\boldsymbol{x}, \boldsymbol{y} \in G$. Let $(G, G)$ denote the subgroup of $G$ generated by all commutators of $G$. If one defines $G_{1}=(G, G), G_{2}=\left(G_{1}, G_{1}\right), \ldots, G_{k}=\left(G_{k-1}, G_{k-1}\right)$ then $G$ is called solvable if $G_{k}=\mathbf{0}$ for some integer $k$. The Lie algebra of $G_{p}$ is $\mathfrak{g}_{p}$ and $G$ is solvable if and only if $\mathfrak{g}$ is solvable. In a solvable Lie algebra of dimension three, it can be shown that there are basis vectors $\boldsymbol{f}_{1}, \boldsymbol{f}_{2}, \boldsymbol{f}_{3}$ of $\mathbb{R}^{3}$ such that

$$
\left[\boldsymbol{f}_{1}, \boldsymbol{f}_{2}\right]=\mathbf{0}, \quad\left[\boldsymbol{f}_{1}, \boldsymbol{f}_{3}\right]=\alpha \boldsymbol{f}_{1}+\beta \boldsymbol{f}_{2}, \quad\left[\boldsymbol{f}_{2}, \boldsymbol{f}_{3}\right]=\gamma \boldsymbol{f}_{1}+\delta \boldsymbol{f}_{2},
$$

where $\alpha, \beta, \gamma, \delta \in \mathbb{R}$ and $\alpha \delta-\beta \gamma \neq 0$.

Notice that if (4.1) holds then $\mathfrak{g}_{3}=\mathbf{0}$ so that $G_{3}=\mathbf{0}$. So all commutators of elements of $G$ commute with each other. 
4.1 The continuous Lie group $S_{1}$

According to Auslander et al. [1] (subject to some technical conditions), there are only three classes of three-dimensional, non-abelian Lie groups with nontrivial discrete subgroups. In this paper we focus on $S_{1}$, one of the two nonisomorphic classes of connected, simply connected, non-compact, three-dimensional solvable groups with a discrete subgroup $D$. We define this group below and give the form of the corresponding dislocation density tensor. Further details regarding derivation of facts about $S_{1}$ that we state here can be found in Nicks and Parry [19].

4.2 Definition and basic properties of $S_{1}$

Group elements $\boldsymbol{x} \in S_{1}$ are identified with points of $\mathbb{R}^{3}$ by expressing them as $\boldsymbol{x}=x_{i} \boldsymbol{e}_{i}$ with respect to some basis $\boldsymbol{e}_{1}, \boldsymbol{e}_{2}, \boldsymbol{e}_{3}$. Auslander et al. [1] choose to represent the elements as $4 \times 4$ matrices (still parameterised by $\left(x_{1}, x_{2}, x_{3}\right)$ ) and these matrix representations form an isomorphic group $S_{m}$ where the matrix representation of $\boldsymbol{x} \in S_{1}$ is $r_{m}(\boldsymbol{x}) \in S_{m}$, defined by

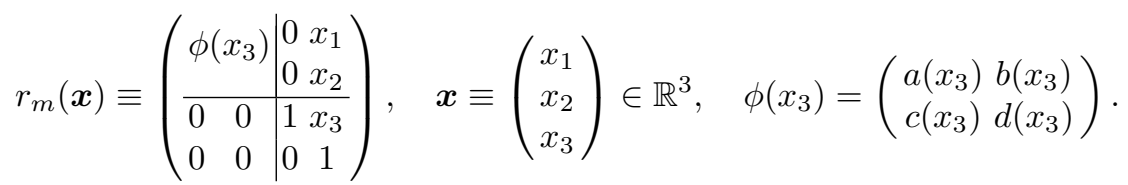

In $(4.2), \phi\left(x_{3}\right) \in S L_{2}(\mathbb{R}), \phi(1) \in S L_{2}(\mathbb{Z})$ and $\left\{\phi\left(x_{3}\right): x_{3} \in \mathbb{R}\right\}$ is a one parameter subgroup of the unimodular group. This implies that

$$
\phi(x) \phi(y)=\phi(x+y), \quad x, y, \in \mathbb{R},
$$

and hence $\phi(0)=\mathbb{I}_{2}$, the $2 \times 2$ identity matrix. The one parameter subgroups of $S L_{2}(\mathbb{R})$ which have $\phi(1) \in S L_{2}(\mathbb{Z})$ fall into two classes depending on the eigenvalues of $\phi(1)$. Let us define

$$
\phi(1) \equiv \theta=\left(\begin{array}{ll}
a(1) & b(1) \\
c(1) & d(1)
\end{array}\right)=\left(\begin{array}{ll}
a & b \\
c & d
\end{array}\right), \quad a, b, c, d \in \mathbb{Z}, \quad a d-b c=1 .
$$

The eigenvalues, $\lambda$, of $\theta$ are roots of the characteristic equation

$$
P(\lambda)=\lambda^{2}-\operatorname{tr}(\theta) \lambda+1
$$

and are therefore given by $\lambda, 1 / \lambda$ where $\lambda=\frac{1}{2}\left(a+d+\sqrt{(a+d)^{2}-4}\right)$. If $\operatorname{tr}(\theta)>2$ then the eigenvalues of $\theta$ are real and distinct and the group of matrices of the form $r_{m}(\boldsymbol{x})$ is isomorphic to $S_{1}$. If $\operatorname{tr}(\theta) \in\{-2,-1,0,1\}$ then the eigenvalues of $\theta$ are a complex conjugate pair and the group of matrices of the form $r_{m}(\boldsymbol{x})$ is isomorphic to another class of solvable group with discrete subgroups (this group $S_{2}$ shall be considered elsewhere). Moreover, if $\operatorname{tr}(\theta)=$ -2 then $\theta \equiv-\mathbb{I}_{2}$. If $\operatorname{tr}(\theta)=2$ then the group of matrices of the form $r_{m}(\boldsymbol{x})$ is 
isomorphic to a nilpotent Lie group. For other values of $\operatorname{tr}(\theta), \theta$ cannot lie on a one parameter subgroup of $S L_{2}(\mathbb{R})$ (see Auslander [1]).

Let us also define

$$
\phi^{\prime}(0)=\mathcal{A}=\left(\begin{array}{ll}
a^{\prime}(0) & b^{\prime}(0) \\
c^{\prime}(0) & d^{\prime}(0)
\end{array}\right), \quad \text { where }{ }^{\prime} \text { denotes } \frac{d}{d x} .
$$

Differentiating (4.3) with respect to $y$ and putting $y=0$ and similarly for $x$ we see that the one parameter subgroup $\phi(x)$ satisfies

$$
\phi^{\prime}(x)=\phi(x) \mathcal{A}=\mathcal{A} \phi(x) \quad \Rightarrow \quad \phi(x)=\mathrm{e}^{\mathcal{A} x} \equiv \sum_{j=0}^{\infty} \mathcal{A}^{j} \frac{x^{j}}{j !} .
$$

Moreover, because $\phi(x) \in S L_{2}(\mathbb{R}), a(x) d(x)-b(x) c(x)=1$. Differentiating this relation and setting $x=0$ we see that $a^{\prime}(0)+d^{\prime}(0)=0$ and hence

$$
\mathcal{A}^{2}=-\operatorname{det}(\mathcal{A}) \mathbb{I}_{2}
$$

For any matrix $\mathcal{A}$ satisfying (4.8),

$$
\mathrm{e}^{\mathcal{A}}= \begin{cases}(\cosh k) \mathbb{I}_{2}+\left(\frac{\sinh k}{k}\right) \mathcal{A}, & \text { if } \operatorname{det}(\mathcal{A})>0, \quad k \equiv \sqrt{-\operatorname{det}(\mathcal{A})} \\ (\cos k) \mathbb{I}_{2}+\left(\frac{\sin k}{k}\right) \mathcal{A}, & \text { if } \operatorname{det}(\mathcal{A})>0, \quad k \equiv \sqrt{\operatorname{det}(\mathcal{A})} \\ \mathbb{I}_{2}+\mathcal{A}, & \text { if } \operatorname{det}(\mathcal{A})=0 ;\end{cases}
$$

and thus, since $\operatorname{tr}(\mathcal{A})=0$,

$$
a+d=\operatorname{tr} e^{\mathcal{A}}=\left\{\begin{array}{ll}
2 \cosh k, & \text { if } \operatorname{det}(\mathcal{A})<0 ; \\
2 \cos k, & \text { if } \operatorname{det}(\mathcal{A})>0 ; \\
2, & \text { if } \operatorname{det}(\mathcal{A})=0,
\end{array} \quad \text { where } k=\sqrt{|\operatorname{det}(\mathcal{A})|} .\right.
$$

We are concerned with that case where $\operatorname{tr}(\theta)>2$ so that the corresponding $\mathcal{A}$ has $\operatorname{det}(\mathcal{A})=-k^{2}<0$. From (4.9), (4.10) one can then see that when $\operatorname{tr}(\theta)>2$

$$
\mathcal{A}=\frac{k}{\sinh k}\left(\begin{array}{cc}
\frac{1}{2}(a-d) & b \\
c & -\frac{1}{2}(a-d)
\end{array}\right) .
$$

Notice that $b$ and $c$ are non zero, in $\mathcal{A}$ and $\theta$, since if either were zero, this would imply that $a d=1$ and hence $a=d= \pm 1$ since $a, d \in \mathbb{Z}$, implying that $\operatorname{tr}(\theta)= \pm 2$. 
4.3 The Lie algebra $\mathfrak{s}_{1}$ and the corresponding dislocation density tensor $S$

Group composition in $S_{m}$ is matrix multiplication, thus if $\boldsymbol{\psi}$ is the group composition function in $S_{1}$ then from the fact that $r_{m}(\boldsymbol{x}) r_{m}(\boldsymbol{y})=r_{m}(\boldsymbol{\psi}(\boldsymbol{x}, \boldsymbol{y}))$ for $\boldsymbol{x}, \boldsymbol{y} \in S_{1}$ one computes that

$$
\boldsymbol{\psi}(\boldsymbol{x}, \boldsymbol{y})=\boldsymbol{x}+\left(a\left(x_{3}\right) y_{1}+b\left(x_{3}\right) y_{2}\right) \boldsymbol{e}_{1}+\left(c\left(x_{3}\right) y_{1}+d\left(x_{3}\right) y_{2}\right) \boldsymbol{e}_{2}+y_{3} \boldsymbol{e}_{3}
$$

where $\boldsymbol{x}=x_{i} \boldsymbol{e}_{i}, \boldsymbol{y}=y_{i} \boldsymbol{e}_{i}$ and $\left\{\boldsymbol{e}_{1}, \boldsymbol{e}_{2}, \boldsymbol{e}_{3}\right\}$ is a basis of $\mathbb{R}^{3}$.

One calculates that the Lie bracket on $\mathfrak{s}_{1}$, the Lie algebra of $S_{1}$, is given by

$$
\begin{aligned}
& {[\boldsymbol{x}, \boldsymbol{y}]=} \\
& \left(a^{\prime}(0) \boldsymbol{x} \wedge \boldsymbol{y} \cdot \boldsymbol{e}_{2}-b^{\prime}(0) \boldsymbol{x} \wedge \boldsymbol{y} \cdot \boldsymbol{e}_{1}\right) \boldsymbol{e}_{1}+\left(c^{\prime}(0) \boldsymbol{x} \wedge \boldsymbol{y} \cdot \boldsymbol{e}_{2}-d^{\prime}(0) \boldsymbol{x} \wedge \boldsymbol{y} \cdot \boldsymbol{e}_{1}\right) \boldsymbol{e}_{2} .
\end{aligned}
$$

In particular

$$
\left[\boldsymbol{e}_{1}, \boldsymbol{e}_{2}\right]=\mathbf{0}, \quad\left[\boldsymbol{e}_{1}, \boldsymbol{e}_{3}\right]=-a^{\prime}(0) \boldsymbol{e}_{1}-c^{\prime}(0) \boldsymbol{e}_{2}, \quad\left[\boldsymbol{e}_{2}, \boldsymbol{e}_{3}\right]=-b^{\prime}(0) \boldsymbol{e}_{1}-d^{\prime}(0) \boldsymbol{e}_{2}
$$

One can also compute that

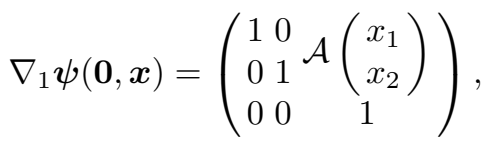

and this gives that the right invariant lattice vector fields $\boldsymbol{\ell}_{a}(\boldsymbol{x}) \equiv \nabla_{1} \boldsymbol{\psi}(\mathbf{0}, \boldsymbol{x}) \boldsymbol{e}_{a}$ are given by

$$
\begin{aligned}
& \boldsymbol{\ell}_{1}(\boldsymbol{x})=\boldsymbol{e}_{1}, \boldsymbol{\ell}_{2}(\boldsymbol{x})=\boldsymbol{e}_{2}, \\
& \boldsymbol{\ell}_{3}(\boldsymbol{x})=\left(a^{\prime}(0) x_{1}+b^{\prime}(0) x_{2}\right) \boldsymbol{e}_{1}+\left(c^{\prime}(0) x_{1}+d^{\prime}(0) x_{2}\right) \boldsymbol{e}_{2}+\boldsymbol{e}_{3} .
\end{aligned}
$$

The dual lattice fields $\boldsymbol{d}_{a}(\boldsymbol{x})$ are

$$
\begin{aligned}
& \boldsymbol{d}_{1}(\boldsymbol{x})=\boldsymbol{e}_{1}-\left(a^{\prime}(0) x_{1}+b^{\prime}(0)\right) \boldsymbol{e}_{3}, \\
& \boldsymbol{d}_{2}(\boldsymbol{x})=\boldsymbol{e}_{2}-\left(c^{\prime}(0) x_{1}+d^{\prime}(0) x_{2}\right) \boldsymbol{e}_{3}, \boldsymbol{d}_{3}(\boldsymbol{x})=\boldsymbol{e}_{3} .
\end{aligned}
$$

Hence the lattice components of the dislocation density tensor are

$$
\left(\frac{\nabla \wedge \boldsymbol{d}_{a} \cdot \boldsymbol{d}_{b}}{\boldsymbol{d}_{1} \cdot \boldsymbol{d}_{2} \wedge \boldsymbol{d}_{3}}\right)=\left(\begin{array}{ccc}
-b^{\prime}(0) & a^{\prime}(0) & 0 \\
a^{\prime}(0) & c^{\prime}(0) & 0 \\
0 & 0 & 0
\end{array}\right),
$$

recalling that $a^{\prime}(0)=-d^{\prime}(0)$. In particular the dislocation density tensor is rank 2 , symmetric and defined by the matrix $\mathcal{A}$.

\subsection{Automorphisms of $S_{1}$ and $\mathfrak{s}_{1}$}

Here we find the Lie algebra automorphisms and compute the group automorphisms of $S_{1}$ via exponentiation (noting that the exponential is surjective, here). 
4.4.1 Automorphisms of $\mathfrak{s}_{1}$

Let $\left\{\boldsymbol{e}_{1}, \boldsymbol{e}_{2}, \boldsymbol{e}_{3}\right\}$ be the basis of $\mathbb{R}^{3}$ such that (4.12) holds with $a^{\prime}(0), b^{\prime}(0), c^{\prime}(0), d^{\prime}(0)$ the entries in the matrix $\mathcal{A}$ as given in (4.6) where $\operatorname{det}(\mathcal{A})=-k^{2}$. The eigenvalues of $\mathcal{A}$ are $\pm k$ and since $\mathcal{A}$ does not have any zero off diagonal entries by the last remark of of section $4.2, \mathcal{A}$ can be diagonalized. This diagonalization is equivalent to a change of basis in the Lie algebra $\mathfrak{s}_{1}$ to a basis $\left\{\boldsymbol{f}_{1}, \boldsymbol{f}_{2}, \boldsymbol{f}_{3}\right\}$ which satisfies

$$
\left[\boldsymbol{f}_{1}, \boldsymbol{f}_{2}\right]=\mathbf{0}, \quad\left[\boldsymbol{f}_{3}, \boldsymbol{f}_{1}\right]=k \boldsymbol{f}_{1}, \quad\left[\boldsymbol{f}_{3}, \boldsymbol{f}_{2}\right]=-k \boldsymbol{f}_{2} .
$$

One such basis is given by $\boldsymbol{f}_{i}=M_{i j} \boldsymbol{e}_{j}$ where

$$
M:=\left(\begin{array}{ccc}
-b^{\prime}(0) & a^{\prime}(0)-k & 0 \\
-b^{\prime}(0) & a^{\prime}(0)+k & 0 \\
0 & 0 & 1
\end{array}\right) .
$$

With respect to the basis $\left\{\boldsymbol{f}_{1}, \boldsymbol{f}_{2}, \boldsymbol{f}_{3}\right\}$ the structure constants of $\mathfrak{s}_{1}$ are given by $C_{i j k}=k\left(\delta_{i 1} \epsilon_{2 j k}+\delta_{i 2} \epsilon_{1 j k}\right)$ and using (1.23) with $\mathfrak{g}=\mathfrak{g}^{\prime}=\mathfrak{s}_{1}$ one computes that a linear transformation $L$ is an automorphism of $\mathfrak{s}_{1}$ with respect to the basis $\left\{\boldsymbol{f}_{1}, \boldsymbol{f}_{2}, \boldsymbol{f}_{3}\right\}$ if $L$ has the form (c.f. [12])

$$
L=\left(\begin{array}{ccc}
0 & 1 & 0 \\
1 & 0 & 0 \\
0 & 0 & -1
\end{array}\right)^{\epsilon}\left(\begin{array}{ccc}
\alpha & 0 & \gamma \\
0 & \beta & \delta \\
0 & 0 & 1
\end{array}\right), \quad \epsilon \in\{0,1\}, \alpha, \beta, \gamma, \delta \in \mathbb{R}, \quad \alpha \beta \neq 0 .
$$

With respect to the basis $\left\{\boldsymbol{e}_{1}, \boldsymbol{e}_{2}, \boldsymbol{e}_{3}\right\}$ the automorphisms of $\mathfrak{s}_{1}$ are given by $M^{T} L M^{-T}$ where $T$ denotes transpose.

\subsubsection{Automorphisms of $S_{1}$}

The elements of the Lie algebra $\mathfrak{s}_{1}$ and the Lie group $S_{1}$ are linked by the exponential mapping $\mathrm{e}^{(\cdot)}: \mathfrak{s}_{1} \rightarrow S_{1}$. We compute this mapping with respect to the basis $\boldsymbol{f}_{1}, \boldsymbol{f}_{2}, \boldsymbol{f}_{3}$. When changing basis from $\left\{\boldsymbol{e}_{1}, \boldsymbol{e}_{2}, \boldsymbol{e}_{3}\right\}$ to $\left\{\boldsymbol{f}_{1}, \boldsymbol{f}_{2}, \boldsymbol{f}_{3}\right\}$ the matrix $\mathcal{A}$ changes to $\mathcal{B}=\left(\begin{array}{cc}k & 0 \\ 0 & -k\end{array}\right)$ and, with respect to the basis $\left\{\boldsymbol{f}_{1}, \boldsymbol{f}_{2}, \boldsymbol{f}_{3}\right\}$, $\phi(x)=\mathrm{e}^{\mathcal{B} x}$. Thus with respect to the basis $\left\{\boldsymbol{f}_{1}, \boldsymbol{f}_{2}, \boldsymbol{f}_{3}\right\}$ the Lie group composition function in $S_{1}$ is given by

$$
\boldsymbol{\psi}(\boldsymbol{u}, \boldsymbol{v})=\boldsymbol{u}+\left(v_{1} \mathrm{e}^{k u_{3}}\right) \boldsymbol{f}_{1}+\left(v_{2} \mathrm{e}^{-k u_{3}}\right) \boldsymbol{f}_{2}+v_{3} \boldsymbol{f}_{3}
$$

where $\boldsymbol{u}=u_{i} \boldsymbol{f}_{i}, \boldsymbol{v}=v_{i} \boldsymbol{f}_{i}$. By computing the lattice vector fields $\nabla_{1} \boldsymbol{\psi}(\mathbf{0}, \boldsymbol{u}) \boldsymbol{f}_{a}$ one finds that the exponential mapping $\mathrm{e}^{(\cdot)}: \mathfrak{s}_{1} \rightarrow S_{1}$ with respect to the basis $\left\{\boldsymbol{f}_{1}, \boldsymbol{f}_{2}, \boldsymbol{f}_{3}\right\}$ is given by

$$
\mathrm{e}^{(\boldsymbol{u})}=\left(\begin{array}{c}
F\left(\mathcal{B} u_{3}\right) \\
u_{3}
\end{array}\left(\begin{array}{l}
u_{1} \\
u_{2}
\end{array}\right)\right) \quad \text { where } \boldsymbol{u}=u_{i} \boldsymbol{f}_{i}=\left(u_{1}, u_{2}, u_{3}\right)^{T},
$$


and

$$
\begin{aligned}
F\left(\mathcal{B} u_{3}\right) & =\sum_{j=0}^{\infty} \frac{\left(\mathcal{B} u_{3}\right)^{j}}{(j+1) !} \\
& =\left(\frac{\sinh \left(k u_{3}\right)}{k u_{3}}\right) \mathbb{I}_{2}+\left(\frac{\cosh \left(k u_{3}\right)-1}{\left(k u_{3}\right)^{2}}\right) \mathcal{B} u_{3}=\left(\begin{array}{cc}
\frac{\mathrm{e}^{k u_{3}}-1}{k u_{3}} & 0 \\
0 & \frac{\mathrm{e}^{-k u_{3}-1}}{-k u_{3}}
\end{array}\right) .
\end{aligned}
$$

Note that this mapping is a homeomorphism so has a well defined inverse $\ln (\cdot) \equiv\left(\mathrm{e}^{(\cdot)}\right)^{-1}: S_{1} \rightarrow \mathfrak{s}_{1}$ given by

$$
\ln (\boldsymbol{u})=\left(\begin{array}{c}
\left.\left(F\left(\mathcal{B} u_{3}\right)\right)^{-1}\left(\begin{array}{l}
u_{1} \\
u_{2}
\end{array}\right)\right) \\
u_{3}
\end{array}\right)
$$

where

$$
\left(F\left(\mathcal{B} u_{3}\right)\right)^{-1}=\frac{u_{3} k}{2} \operatorname{coth}\left(\frac{u_{3} k}{2}\right) \mathbb{I}_{2}-\frac{1}{2} \mathcal{B} u_{3}=\left(\begin{array}{cc}
\frac{k u_{3}}{\mathrm{e}^{k u_{3}-1}} & 0 \\
0 & \frac{-k u_{3}}{\mathrm{e}^{-k u_{3}-1}}
\end{array}\right) .
$$

Recall from section 2 that if $\phi: S_{1} \rightarrow S_{1}$ is a Lie group automorphism then $\nabla \boldsymbol{\phi}(\mathbf{0})=L$ is a Lie algebra automorphism. Also

$$
\phi\left(\mathrm{e}^{(\boldsymbol{u})}\right)=\mathrm{e}^{(L \boldsymbol{u})} \quad \boldsymbol{u} \in \mathfrak{s}_{1} .
$$

Let $\boldsymbol{x}=\mathrm{e}^{(\boldsymbol{u})}$ so that $\boldsymbol{u}=\ln (\boldsymbol{x})$. Then

$$
\begin{gathered}
L \boldsymbol{u}=\left(L^{\prime}(\epsilon)\left(\begin{array}{l}
u_{1} \\
u_{2}
\end{array}\right)+u_{3}\left(\begin{array}{l}
l_{1}(\epsilon) \\
l_{2}(\epsilon)
\end{array}\right)\right), \\
\mathrm{e}^{(L \boldsymbol{u})}=\left(\begin{array}{c}
F\left(\mathcal{B} \xi u_{3}\right) \\
\left(L^{\prime}(\epsilon)\left(\begin{array}{c}
u_{1} \\
u_{2}
\end{array}\right)+u_{3}\left(\begin{array}{l}
l_{1}(\epsilon) \\
l_{2}(\epsilon)
\end{array}\right)\right) \\
\xi u_{3}
\end{array}\right)
\end{gathered}
$$

where from $(4.20)$

$$
L^{\prime}(\epsilon)=\left(\begin{array}{ll}
0 & 1 \\
1 & 0
\end{array}\right)^{\epsilon}\left(\begin{array}{ll}
\alpha & 0 \\
0 & \beta
\end{array}\right), \quad\left(\begin{array}{l}
l_{1}(\epsilon) \\
l_{2}(\epsilon)
\end{array}\right)=\left(\begin{array}{ll}
0 & 1 \\
1 & 0
\end{array}\right)^{\epsilon}\left(\begin{array}{l}
\gamma \\
\delta
\end{array}\right), \quad \xi=(-1)^{\epsilon} .
$$

Therefore the automorphisms of $S_{1}$ with respect to the basis $\left\{\boldsymbol{f}_{1}, \boldsymbol{f}_{2}, \boldsymbol{f}_{3}\right\}$ are given by

$$
\begin{array}{r}
\phi(\boldsymbol{x})=\left(\begin{array}{c}
F\left(\mathcal{B} \xi x_{3}\right)\left(L^{\prime}(\epsilon) F\left(\mathcal{B} x_{3}\right)^{-1}\left(\begin{array}{l}
x_{1} \\
x_{2}
\end{array}\right)+x_{3}\left(\begin{array}{l}
l_{1}(\epsilon) \\
l_{2}(\epsilon)
\end{array}\right)\right) \\
\xi x_{3}
\end{array}\right) \\
=\left\{\begin{array}{c}
\left(\begin{array}{c}
\alpha x_{1}+\frac{\gamma}{k}\left(\mathrm{e}^{k x_{3}}-1\right) \\
\beta x_{2}-\frac{\delta}{k}\left(\mathrm{e}^{-k x_{3}}-1\right) \\
x_{3}
\end{array}\right) \text { when } \epsilon=0 \\
\left(\begin{array}{c}
\beta x_{2}-\frac{\delta}{k}\left(\mathrm{e}^{-k x_{3}}-1\right) \\
\alpha x_{1}+\frac{\gamma}{k}\left(\mathrm{e}^{k x_{3}}-1\right) \\
-x_{3}
\end{array}\right) \text { when } \epsilon=1
\end{array}\right.
\end{array}
$$


4.5 The Lie groups $\operatorname{Aut}\left(\mathfrak{s}_{1}\right), \operatorname{Aut}\left(S_{1}\right)$

In the last section we saw that with respect to the basis $\left\{\boldsymbol{f}_{1}, \boldsymbol{f}_{2}, \boldsymbol{f}_{3}\right\}$ the automorphisms of $\mathfrak{s}_{1}$ have the form (4.20). These automorphisms form a Lie group $\operatorname{Aut}\left(\mathfrak{s}_{1}\right)$ where

$$
\begin{aligned}
& \operatorname{Aut}\left(\mathfrak{s}_{1}\right)= \\
& \left\{L: \mathfrak{s}_{1} \rightarrow \mathfrak{s}_{1}: L=\left(\begin{array}{ccc}
0 & 1 & 0 \\
1 & 0 & 0 \\
0 & 0 & -1
\end{array}\right)^{\epsilon}\left(\begin{array}{ccc}
\alpha & 0 & \gamma \\
0 & \beta & \delta \\
0 & 0 & 1
\end{array}\right), \epsilon \in\{0,1\}, \alpha, \beta, \gamma, \delta \in \mathbb{R}, \alpha \beta \neq 0 .\right\}
\end{aligned}
$$

Define the following subgroups of $\operatorname{Aut}\left(\mathfrak{s}_{1}\right)$ :

$$
\begin{gathered}
P:=\left\{L \in \operatorname{Aut}\left(\mathfrak{s}_{1}\right): L=\left(\begin{array}{ccc}
0 & 1 & 0 \\
1 & 0 & 0 \\
0 & 0 & -1
\end{array}\right)^{\epsilon}, \epsilon \in\{0,1\}\right\} \\
R:=\left\{L \in \operatorname{Aut}\left(\mathfrak{s}_{1}\right): L=\left(\begin{array}{lll}
\alpha & 0 & \gamma \\
0 & \beta & \delta \\
0 & 0 & 1
\end{array}\right), \quad \alpha, \beta, \gamma, \delta \in \mathbb{R}, \quad \alpha \beta \neq 0\right\} .
\end{gathered}
$$

Then, noting that $R$ is normal in $\operatorname{Aut}\left(\mathfrak{s}_{1}\right)$ and $\operatorname{Aut}\left(\mathfrak{s}_{1}\right)=P R$, if $L \in \operatorname{Aut}\left(\mathfrak{s}_{1}\right)$ then $L$ can be written uniquely as a product of an element of $P$ and an element of $R$. Also define the following subgroups of $R$ :

$$
\begin{gathered}
S:=\left\{L \in \operatorname{Aut}\left(\mathfrak{s}_{1}\right): L=\left(\begin{array}{lll}
\alpha & 0 & 0 \\
0 & \beta & 0 \\
0 & 0 & 1
\end{array}\right), \quad \alpha, \beta \in \mathbb{R}, \quad \alpha \beta \neq 0\right\}, \\
T:=\left\{L \in \operatorname{Aut}\left(\mathfrak{s}_{1}\right): L=\left(\begin{array}{lll}
1 & 0 & \gamma \\
0 & 1 & \delta \\
0 & 0 & 1
\end{array}\right), \quad \gamma, \delta \in \mathbb{R}\right\} .
\end{gathered}
$$

Then $T$ is normal in $R$ and $R=S T$ so that any $L \in \operatorname{Aut}\left(\mathfrak{s}_{1}\right)$ can be uniquely written as a product of an element $\mathrm{P}$, an element of $\mathrm{S}$ and an element of $T$ or $\operatorname{Aut}\left(\mathfrak{s}_{1}\right)=P S T$, which we might loosely term a rotation, a pure strain, a shear, respectively.

There is a one to one correspondence between the automorphisms of $\mathfrak{s}_{1}$ and the automorphisms of $S_{1}$, in fact there is an isomorphism $\boldsymbol{\mu}:$ Aut $\left(\mathfrak{s}_{1}\right) \rightarrow$ $\operatorname{Aut}\left(S_{1}\right)$, and the Lie group automorphism $\boldsymbol{\mu}(L)=\phi_{L}$ corresponding to the Lie algebra automorphism $L=$ pst, $p \in P, s \in S, t \in T$, can be uniquely decomposed as

$$
\boldsymbol{\mu}(L)=\boldsymbol{\mu}(p) \circ \boldsymbol{\mu}(s) \circ \boldsymbol{\mu}(t)
$$

where $\boldsymbol{\mu}(p) \in \boldsymbol{\mu}(P)$ and $\boldsymbol{\mu}(P)$ is a subgroup of $\operatorname{Aut}\left(S_{1}\right)$, and so on. 


\section{Discrete subgroups of $S_{1}$ and their symmetries}

In this section we find the discrete subgroups $D$ of the continuous solvable Lie group $S_{1}$. We also investigate how different sets of generators of $D$ are related, one to the other.

\subsection{The discrete subgroups $D$ of $S_{1}$}

According to Auslander et al. [1], when $\theta \in S L_{2}(\mathbb{Z})$ is such that $\operatorname{tr}(\theta)>2$ the discrete subgroups $D \subset S_{1}$ are isomorphic (via $r_{m}(\cdot)$ defined in (4.2)) to a discrete subgroup $D_{m} \subset S_{m}$ and $D_{m}$ is generated by three elements

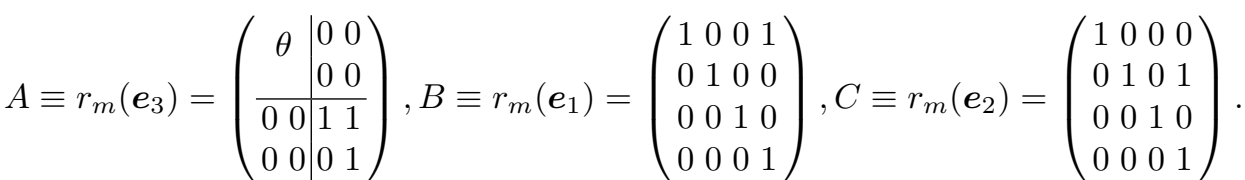

(Here again we work with respect to the basis $\left\{\boldsymbol{e}_{1}, \boldsymbol{e}_{2}, \boldsymbol{e}_{3}\right\}$.) Recall that the commutator of elements $\boldsymbol{x}, \boldsymbol{y} \in S_{1}$, is denoted $(\boldsymbol{x}, \boldsymbol{y})=\boldsymbol{x}^{-1} \boldsymbol{y}^{-1} \boldsymbol{x} \boldsymbol{y}$ where we write $\boldsymbol{x} \boldsymbol{y}=\boldsymbol{\psi}(\boldsymbol{x}, \boldsymbol{y})$. Similarly, let $(X, Y)=X^{-1} Y^{-1} X Y$ denote the commutator of elements $X, Y \in D_{m}$. One can then compute that

$$
(A, B)=B^{1-d} C^{c}, \quad(A, C)=B^{b} C^{1-a}, \quad(B, C)=\mathbb{I}_{4},
$$

where $\mathbb{I}_{4}$ is the identity element in $D_{m}$. From this one can see that any element of $D_{m}$ can be expressed as a product of the form

$$
d_{m}=A^{\alpha_{1}} B^{\beta_{1}} C^{\gamma_{1}} A^{\alpha_{2}} B^{\beta_{2}} C^{\gamma_{2}} \ldots A^{\alpha_{r}} B^{\beta_{r}} C^{\gamma_{r}}=A^{\alpha_{1}+\cdots \alpha_{r}} B^{M} C^{N}
$$

for some $M, N \in \mathbb{Z}$ where $\alpha_{i}, \beta_{i}, \gamma_{i} \in \mathbb{Z}, i=1,2, \ldots, r$ and $r \in \mathbb{Z}$. A general element $d_{m}=A^{Q} B^{M} C^{N} \in D_{m}, Q, M, N \in \mathbb{Z}$ has the representation

$$
d_{m}=\left(\begin{array}{c|ccc}
\theta^{Q} & \begin{array}{l}
0 \\
\end{array} & \theta^{Q}\left(\begin{array}{c}
M \\
N
\end{array}\right) \\
\hline 0 & 0 & 1 & Q \\
0 & 0 & 0 & 1
\end{array}\right)=r_{m}(\boldsymbol{x}), \quad \text { where } \boldsymbol{x}=\left(\begin{array}{c}
\theta^{Q}\left(\begin{array}{c}
M \\
N
\end{array}\right) \\
Q
\end{array}\right) \in S_{1},
$$

with respect to the basis $\left\{\boldsymbol{e}_{1}, \boldsymbol{e}_{2}, \boldsymbol{e}_{3}\right\}$.

It is then clear that since $\theta \in S L_{2}(\mathbb{Z}), r_{m}^{-1}\left(D_{m}\right)=D=\left(\mathbb{Z}^{3}, \boldsymbol{\psi}\right)$. Thus the discrete structures which are the analogues of perfect lattices $L$ in this case are the cubic lattice $\mathbb{Z}^{3}$ with group multiplication $\boldsymbol{\psi}$ given by (4.12).

\subsection{Free substitutions and generators of $D$}

The set of symmetries of a discrete subgroup $D \subset S_{1}$ comprises of the choices of three elements $\boldsymbol{g}_{1}, \boldsymbol{g}_{2}, \boldsymbol{g}_{3} \in D$ such that the group generated by these elements, denoted $G=g p\left(\boldsymbol{g}_{1}, \boldsymbol{g}_{2}, \boldsymbol{g}_{3}\right)$, equals $D$. These are the changes of generators which preserves the integer lattice $\mathbb{Z}^{3}$. In this case, it turns out that the 
conditions on $\boldsymbol{g}_{1}, \boldsymbol{g}_{2}, \boldsymbol{g}_{3}$ that are necessary and sufficient that $G=D$ are the same as those that are necessary and sufficient that the commutator subgroups $G^{\prime}$ and $D^{\prime}$ are equal. In section 5.2.2 we state these conditions mostly without proof. However we provide the proof of Lemma 1 in order to correct a minor flaw in the proof given in [19]. Details of the proof of other statements below can be found in [19].

We first recall in the next section the connection between free substitutions and changes of generators of a free group, following Magnus, Karrass and Solitar [17].

\subsubsection{Free substitutions}

Let $X$ be a set (which we will later take to be a set of generators of the discrete subgroup $D$ ) and let $F(X)$ be the free group on $X$ so that $F(X)$ consists of all words in the elements of $X$ and their inverses. Suppose, for example, that $X=\left\{x_{1}, x_{2}, x_{3}\right\}$ then the words of $F(X)$ have the form

$w=x_{1}^{\alpha_{1}} x_{2}^{\beta_{1}} x_{3}^{\gamma_{1}} x_{1}^{\alpha_{2}} x_{2}^{\beta_{2}} x_{3}^{\gamma_{2}} \cdots x_{1}^{\alpha_{r}} x_{2}^{\beta_{r}} x_{3}^{\gamma_{r}} \quad r \in \mathbb{Z}, \alpha_{i}, \beta_{i}, \gamma_{i} \in \mathbb{Z}, i=1,2, \ldots, r$.

The group operation in $F(X)$ is juxtaposition of words with terms of the form $x_{i} x_{i}^{-1}, x_{i}^{-1} x_{i}$ cancelled in any product of words. A free substitution of $F(X)$ is a replacement of the elements $x_{1}, x_{2}, x_{3}$ by the words $w_{1}\left(x_{1}, x_{2}, x_{3}\right)$, $w_{2}\left(x_{1}, x_{2}, x_{3}\right), w_{3}\left(x_{1}, x_{2}, x_{3}\right) \in F(X)$ such that these words are also a basis for $F(X)$. This implies that each $x_{i}$ may be written as a word in the $w_{1}, w_{2}, w_{3}$. A free substitution gives rise to a mapping which sends $w\left(x_{1}, x_{2}, x_{3}\right) \in F(X)$ to $w\left(w_{1}\left(x_{1}, x_{2}, x_{3}\right), w_{2}\left(x_{1}, x_{2}, x_{3}\right), w_{3}\left(x_{1}, x_{2}, x_{3}\right)\right) \in F(X)$ and it is a fact that this mapping is an automorphism of $F(X)$. Thus free substitutions represent changes in the set of generators of a free group.

The free substitutions include the following so-called 'elementary Nielsen transformations': permute a pair of generators, replace one generator by the product of that generator with another, replace one generator by its inverse. It is clear that transformations of these three types change one set of generators to another such set (in any finitely generated group), so that successive transformations of this type have the same property.

Note that in our case the discrete subgroup $D$ is not a free group; it has relations given by the known values of the commutators of the generators $A, B, C$ of $D_{m}$ as in (5.2). Let $\boldsymbol{g}_{i m}=r_{m}\left(\boldsymbol{g}_{i}\right)$ for $\boldsymbol{g}_{i} \in D$. Then we may write, in this case, bearing in mind the remarks that precede (5.3)

$$
\begin{aligned}
& \boldsymbol{g}_{1 m}=A^{\alpha_{1}} B^{\beta_{1}} C^{\gamma_{1}}, \\
& \boldsymbol{g}_{2 m}=A^{\alpha_{2}} B^{\beta_{2}} C^{\gamma_{2}}, \\
& \boldsymbol{g}_{3 m}=A^{\alpha_{3}} B^{\beta_{3}} C^{\gamma_{3}},
\end{aligned}
$$

\subsubsection{Generators of $D$}

If $G=g p\left(\boldsymbol{g}_{1}, \boldsymbol{g}_{2}, \boldsymbol{g}_{3}\right)=D$ then $G_{m}=g p\left\{\boldsymbol{g}_{1 m}, \boldsymbol{g}_{2 m}, \boldsymbol{g}_{3 m}\right\}=D_{m}$. Clearly, since $A \in D_{m}$ we must have $A \in G_{m}$ if we are to have $G=D$. This implies that $\operatorname{hcf}\left(\alpha_{1}, \alpha_{2}, \alpha_{3}\right)=1$ and the following lemma holds. 
Lemma 1 Let $\boldsymbol{g}_{1 m}, \boldsymbol{g}_{2 m}, \boldsymbol{g}_{3 m}$ be given by (5.4), let $G_{m}=g p\left(\boldsymbol{g}_{1 m}, \boldsymbol{g}_{2 m}, \boldsymbol{g}_{3 m}\right)$ and suppose that $\operatorname{hcf}\left(\alpha_{1}, \alpha_{2}, \alpha_{3}\right)=1$. Then there is a set of generators of $G_{m}$, denoted $\boldsymbol{g}_{1 m}^{\prime}, \boldsymbol{g}_{2 m}^{\prime}, \boldsymbol{g}_{3 m}^{\prime}$, such that

$\boldsymbol{g}_{1 m}^{\prime}=A B^{\beta_{1}^{\prime}} C^{\gamma_{1}^{\prime}}, \boldsymbol{g}_{2 m}^{\prime}=B^{\beta_{2}^{\prime}} C^{\gamma_{2}^{\prime}}, \boldsymbol{g}_{3 m}^{\prime}=B^{\beta_{3}^{\prime}} C^{\gamma_{3}^{\prime}}, \beta_{i}^{\prime}, \gamma_{i}^{\prime} \in \mathbb{Z}, i=1,2,3$.

Proof Given $\boldsymbol{g}_{m}=A^{\alpha} B^{\beta} C^{\gamma}$ note that $\boldsymbol{g}_{m}^{A}=\alpha$ is well defined because $A^{q} \neq$ $B^{m} C^{n}$ for any $m, n, q \in \mathbb{Z} \backslash\{0\}$. Note that if $P \in S L_{3}(\mathbb{Z})$ with

$$
P=\left(P_{i j}\right) \equiv\left(\begin{array}{lll}
a_{1} & a_{2} & a_{3} \\
b_{1} & b_{2} & b_{3} \\
c_{1} & c_{2} & c_{3}
\end{array}\right), \quad i, j=1,2,3,
$$

and $\left\{\boldsymbol{g}_{1 m}, \boldsymbol{g}_{2 m}, \boldsymbol{g}_{3 m}\right\} P$ is defined to be the set of elements

$$
\left\{\boldsymbol{g}_{1 m}^{a_{1}} \boldsymbol{g}_{2 m}^{b_{1}} \boldsymbol{g}_{3 m}^{c_{1}}, \boldsymbol{g}_{1 m}^{a_{2}} \boldsymbol{g}_{2 m}^{b_{2}} \mathrm{~g}_{3 m}^{c_{2}}, \boldsymbol{g}_{1 m}^{a_{3}} \boldsymbol{g}_{2 m}^{b_{3}} \boldsymbol{g}_{3 m}^{c_{3}}\right\} \equiv\left\{\boldsymbol{g}_{1 m}^{\prime}, \boldsymbol{g}_{2 m}^{\prime}, \boldsymbol{g}_{3 m}^{\prime}\right\}
$$

of $G_{m}$, then $\boldsymbol{g}_{1 m}^{\prime A}=\boldsymbol{g}_{1 m}^{A} a_{1}+\boldsymbol{g}_{2 m}^{A} b_{1}+\boldsymbol{g}_{3 m}^{A} c_{1}$, etc., so $\boldsymbol{g}_{i m}^{\prime A}=\boldsymbol{g}_{j m}^{A} P_{j i}, i=1,2,3$. Now let

$$
R=\left(R_{i j}\right) \equiv\left(\begin{array}{lll}
\bar{a}_{1} & \bar{a}_{2} & \bar{a}_{3} \\
\bar{b}_{1} & \bar{b}_{2} & \bar{b}_{3} \\
\bar{c}_{1} & \bar{c}_{2} & \bar{c}_{3}
\end{array}\right)
$$

and define

$$
\left\{\boldsymbol{g}_{1 m}^{\prime \bar{a}_{1}} \boldsymbol{g}_{2 m}^{\prime \bar{b}_{1}} \boldsymbol{g}_{3 m}^{\prime \bar{c}_{1}}, \boldsymbol{g}_{1 m}^{\prime \bar{a}_{2}} \boldsymbol{g}_{2 m}^{\prime \bar{b}_{2}} \boldsymbol{g}_{3 m}^{\prime \bar{c}_{2}}, \boldsymbol{g}_{1 m}^{\prime \bar{a}_{3}} \boldsymbol{g}_{2 m}^{\bar{b}_{3}} \boldsymbol{g}_{3 m}^{\prime \bar{c}_{3}}\right\} \equiv\left\{\boldsymbol{g}_{1 m}^{\prime \prime}, \boldsymbol{g}_{2 m}^{\prime \prime}, \boldsymbol{g}_{3 m}^{\prime \prime}\right\}
$$

It follows that

$$
\boldsymbol{g}_{i m}^{\prime \prime A}=\boldsymbol{g}_{j m}^{\prime A} R_{j i}=\boldsymbol{g}_{k m}^{A} P_{k j} R_{j i}
$$

whenever

$$
\left\{\boldsymbol{g}_{1 m}^{\prime \prime}, \boldsymbol{g}_{2 m}^{\prime \prime}, \boldsymbol{g}_{3 m}^{\prime \prime}\right\} \equiv\left\{\boldsymbol{g}_{1 m}^{\prime}, \boldsymbol{g}_{2 m}^{\prime}, \boldsymbol{g}_{3 m}^{\prime}\right\} R \equiv\left\{\left\{\boldsymbol{g}_{1 m}, \boldsymbol{g}_{2 m}, \boldsymbol{g}_{3 m}\right\} P\right\} R .
$$

Now note that according to Coxeter and Moser [6, p. 92], any element $P \in$ $S L_{3}(\mathbb{Z})$ is expressible as a product of the following matrices:

$$
\left(\begin{array}{lll}
0 & 1 & 0 \\
0 & 0 & 1 \\
1 & 0 & 0
\end{array}\right),\left(\begin{array}{lll}
1 & 1 & 0 \\
0 & 1 & 0 \\
0 & 0 & 1
\end{array}\right),\left(\begin{array}{ccc}
0 & 1 & 0 \\
-1 & 0 & 0 \\
0 & 0 & 1
\end{array}\right) \text {. }
$$

For each matrix $P^{\prime}$ in the above list, $\left\{\boldsymbol{g}_{1 m}, \boldsymbol{g}_{2 m}, \boldsymbol{g}_{3 m}\right\} P^{\prime}$ generates $G_{m}$, since $\left\{\boldsymbol{g}_{1 m}, \boldsymbol{g}_{2 m}, \boldsymbol{g}_{3 m}\right\} \rightarrow\left\{\boldsymbol{g}_{1 m}, \boldsymbol{g}_{2 m}, \boldsymbol{g}_{3 m}\right\} P^{\prime}$ is a composition of elementary Nielsen transformations. Let $P \in S L_{3}(\mathbb{Z})$ be given by $P=P_{1} P_{2} \cdots P_{n}$ for some $n \in \mathbb{Z}$ where each $P_{i} i=1, \ldots, n$ is a matrix of the list (5.8). Then $\left\{\boldsymbol{g}_{1 m}, \boldsymbol{g}_{2 m}, \boldsymbol{g}_{3 m}\right\} P_{1}$ is a set of generators of $G_{m}$, as are

$$
\begin{aligned}
& \left\{\left\{\boldsymbol{g}_{1 m}, \boldsymbol{g}_{2 m}, \boldsymbol{g}_{3 m}\right\} P_{1}\right\} P_{2}, \ldots,\left\{\left\{\cdots\left\{\left\{\boldsymbol{g}_{1 m}, \boldsymbol{g}_{2 m}, \boldsymbol{g}_{3 m}\right\} P_{1}\right\} P_{2} \cdots\right\} P_{n}\right\} \\
& \quad \equiv\left\{\boldsymbol{g}_{1 m}^{*}, \boldsymbol{g}_{2 m}^{*}, \boldsymbol{g}_{3 m}^{*}\right\} .
\end{aligned}
$$


By successive application of (5.7) one sees that

$$
\boldsymbol{g}_{i m}^{* A}=\boldsymbol{g}_{k m}^{A}\left(P_{1}\right)_{k j_{1}}\left(P_{2}\right)_{j_{1} j_{2}} \cdots\left(P_{n}\right)_{j_{n} i}=\boldsymbol{g}_{k m}^{A} P_{k i} .
$$

Finally let $\left\{\boldsymbol{g}_{1 m}, \boldsymbol{g}_{2 m}, \boldsymbol{g}_{3 m}\right\}$ be as in (5.4) above, so that $\boldsymbol{g}_{i m}^{A}=\alpha_{i}, \quad i=$ $1,2,3$. Since $\operatorname{hcf}\left(\alpha_{1}, \alpha_{2}, \alpha_{3}\right)=1$, one may choose $Q \in S L_{3}(\mathbb{Z})$ with first row $\left(\alpha_{1}, \alpha_{2}, \alpha_{3}\right)$, so that $\left(\alpha_{1}, \alpha_{2}, \alpha_{3}\right) Q^{-1}=(1,0,0)$. Let $P=Q^{-1}$ so that $P \in S L_{3}(\mathbb{Z})$ and $\left\{\boldsymbol{g}_{1 m}^{*}, \boldsymbol{g}_{2 m}^{*}, \boldsymbol{g}_{3 m}^{*}\right\}$ generates $G_{m}$ by the above remarks. Then by $(5.9)$

$$
\boldsymbol{g}_{i m}^{* A}=\boldsymbol{g}_{k m}^{A} P_{k i}=\boldsymbol{g}_{k m}^{A} Q_{k i}^{-1}=\alpha_{k} Q_{k i}^{-1}=\delta_{i 1}, \quad i=1,2,3,
$$

which shows that the set of generators $\left\{\boldsymbol{g}_{1 m}^{*}, \boldsymbol{g}_{2 m}^{*}, \boldsymbol{g}_{3 m}^{*}\right\}$ has the required form.

It is then shown in Nicks and Parry [19] that if one defines $\boldsymbol{\tau}_{1}, \boldsymbol{\tau}_{2}, \boldsymbol{\tau}_{3}, \boldsymbol{\tau}_{4} \in$ $\mathbb{Z}^{2}$ by

$$
\boldsymbol{\tau}_{1}=\left(\begin{array}{c}
\beta_{2}^{\prime} \\
\gamma_{2}^{\prime}
\end{array}\right), \boldsymbol{\tau}_{2}=\left(\begin{array}{c}
\beta_{3}^{\prime} \\
\gamma_{3}^{\prime}
\end{array}\right), \boldsymbol{\tau}_{3}=\theta\left(\begin{array}{c}
\beta_{2}^{\prime} \\
\gamma_{2}^{\prime}
\end{array}\right), \boldsymbol{\tau}_{4}=\theta\left(\begin{array}{c}
\beta_{3}^{\prime} \\
\gamma_{3}^{\prime}
\end{array}\right) ;
$$

where the values of $\beta_{2}^{\prime}, \beta_{3}^{\prime}, \gamma_{2}^{\prime}, \gamma_{3}^{\prime}$ are as in (5.5), then conditions necessary and sufficient that $G=D$ are that

$$
\begin{aligned}
& \operatorname{hcf}\left(\tau_{11}, \tau_{12}, \tau_{13}, \tau_{14}\right)=\operatorname{hcf}\left(\tau_{21}, \tau_{22}, \tau_{23}, \tau_{24}\right)=1 \\
& \operatorname{hcf}\left(\left\{\boldsymbol{\tau}_{i} \wedge \boldsymbol{\tau}_{j} ; i<j, i, j=1,2,3,4\right\}\right)=1,
\end{aligned}
$$

where the components of $\boldsymbol{\tau}_{i}, i=1,2,3,4$, are $\left(\begin{array}{c}\tau_{1 i} \\ \tau_{2 i}\end{array}\right)$. From now on we will drop the primes on the $\beta_{i}^{\prime}, \gamma_{i}^{\prime}$ in the definitions of $\boldsymbol{\tau}_{j}, j=1,2,3,4$.

\section{Automorphisms of discrete subgroups}

Now we consider whether or not the changes of generators of $D$ determined in the previous section extend to automorphisms of that group. By a result of Gorbatsevich [11] if a change of generators of $D \subset S_{1}$ corresponds to an automorphism of $D$ then it extends uniquely to an automorphism of the continuous group $S_{1}$ and thus the automorphisms of $D$ are (restrictions of) elastic deformations of the continuum. At the end of this section we will verify this fact by direct computation. We begin by computing the automorphisms of the discrete subgroups $D$.

\subsection{Changes of generators and automorphisms of $D$}

We use a result of Johnson [13], the 'substitution test' (proposition 3 of chapter 4 ), to prove a result that gives conditions necessary and sufficient in order that a change of generators of $D$ extends to an automorphism of that discrete group 
- the result (Lemma 2) is more general than the analogous result in Nicks and Parry [19].

Denote the generators $A, B, C$ of (4.1) by $A_{1}, A_{2}, A_{3}$. This is a temporary notation convenient for the proof of Lemma 2. (We revert to the original notation once that lemma is proved). Let $\boldsymbol{g}_{1 m}, \boldsymbol{g}_{2 m}, \boldsymbol{g}_{3 m}$ satisfy the conditions of section 5.2.2 so that they, also, generate $D_{m}$. According to (5.4)

$$
\boldsymbol{g}_{i m}=A_{1}^{\alpha_{i}} A_{2}^{\beta_{i}} A_{3}^{\gamma_{i}}, \quad \alpha_{i}, \beta_{i}, \gamma_{i} \in \mathbb{Z}, \quad i=1,2,3,
$$

and since $\left\{\boldsymbol{g}_{1 m}, \boldsymbol{g}_{2 m}, \boldsymbol{g}_{3 m}\right\}$ is a set of generators

$$
A_{i}=\boldsymbol{g}_{1 m}^{a_{i 1}} \boldsymbol{g}_{2 m}^{b_{i 1}} \boldsymbol{g}_{3 m}^{c_{i 1}} \boldsymbol{g}_{1 m}^{a_{i 2}} \boldsymbol{g}_{2 m}^{b_{i 2}} \boldsymbol{g}_{3 m}^{c_{i 2}} \ldots \boldsymbol{g}_{1 m}^{a_{i k}} \boldsymbol{g}_{2 m}^{b_{i k}} \boldsymbol{g}_{3 m}^{c_{i k}}
$$

for some integers $a_{i j}, b_{i j}, c_{i j}, i=1,2,3, j=1,2 \ldots k$, where the integer $k$ may be taken independent of $i$. Denote this last expression briefly by

$$
A_{i}=\boldsymbol{g}_{1 m}^{a_{i 1}} \ldots \boldsymbol{g}_{3 m}^{c_{i k}}, \quad i=1,2,3
$$

Note that from (6.1) and (6.3),

$$
A_{i}=\left(A_{1}^{\alpha_{1}} A_{2}^{\beta_{1}} A_{3}^{\gamma_{1}}\right)^{a_{i 1}} \ldots\left(A_{1}^{\alpha_{3}} A_{2}^{\beta_{3}} A_{3}^{\gamma_{3}}\right)^{c_{i k}}, \quad i=1,2,3,
$$

and that this relation is verified solely by use of the commutator relations (5.2) and the cancellation of group elements and their inverses, when juxtaposed. Also from (6.1) and (6.3)

$$
\boldsymbol{g}_{i m}=\left(\boldsymbol{g}_{1 m}^{a_{11}} \ldots \boldsymbol{g}_{3 m}^{c_{1 k}}\right)^{\alpha_{i}}\left(\boldsymbol{g}_{1 m}^{a_{21}} \ldots \boldsymbol{g}_{3 m}^{c_{2 k}}\right)^{\beta_{i}}\left(\boldsymbol{g}_{1 m}^{a_{31}} \ldots \boldsymbol{g}_{3 m}^{c_{3 k}}\right)^{\gamma_{i}}, \quad i=1,2,3 .
$$

Define mutually inverse mappings $\phi, \tau$ between the sets of generators $\left\{A_{1}, A_{2}, A_{3}\right\},\left\{\boldsymbol{g}_{1 m}, \boldsymbol{g}_{2 m}, \boldsymbol{g}_{3 m}\right\}$ by

$$
\phi\left(A_{i}\right)=\boldsymbol{g}_{i m}, \boldsymbol{\tau}\left(\boldsymbol{g}_{i m}\right)=A_{i}, i=1,2,3 .
$$

We shall refer to relations (5.2), which in the current notation have form

$$
\left(A_{1}, A_{2}\right)=A_{2}^{1-d} A_{3}^{c},\left(A_{1}, A_{3}\right)=A_{2}^{b} A_{3}^{1-a},\left(A_{2}, A_{3}\right)=\mathbb{I}_{4},
$$

as the commutator relations. Assume that

(i) the commutator relations continue to hold when $A_{i}$ is replaced by $\phi\left(A_{i}\right)=$ $\boldsymbol{g}_{i m}, i=1,2,3$.

Then according to the substitution test of Johnson [13], $\phi:\left\{A_{1}, A_{2}, A_{3}\right\} \rightarrow$ $\left\{\boldsymbol{g}_{1 m}, \boldsymbol{g}_{2 m}, \boldsymbol{g}_{3 m}\right\}$ extends to a homomorphism of $D_{m}$ which we denote $\boldsymbol{\phi}^{\prime}$ : $D_{m} \rightarrow D_{m}$. Since $\phi^{\prime}\left(D_{m}\right)$ includes a set of generators of $D_{m}, \phi^{\prime}$ is surjective. Also, since $\phi^{\prime}$ is a homomorphism, from (5.4) we have

$$
\boldsymbol{g}_{i m}=\left(\boldsymbol{g}_{1 m}^{\alpha_{1}} \boldsymbol{g}_{2 m}^{\beta_{1}} \boldsymbol{g}_{3 m}^{\gamma_{1}}\right)^{a_{i 1}} \ldots\left(\boldsymbol{g}_{1 m}^{\alpha_{3}} \boldsymbol{g}_{2 m}^{\beta_{3}} \boldsymbol{g}_{3 m}^{\gamma_{3}}\right)^{c_{i k}}, i=1,2,3
$$

when (i) holds.

When (6.2) is used to express the commutator relations (6.7) in terms of the 
generators $\left\{\boldsymbol{g}_{1 m}, \boldsymbol{g}_{2 m}, \boldsymbol{g}_{3 m}\right\}$ we obtain three relations which we do not write out explicitly, but express symbolically as

$$
\text { C.R. }\left\{\boldsymbol{g}_{1 m}, \boldsymbol{g}_{2 m}, \boldsymbol{g}_{3 m}\right\}=\mathbb{I}_{4} .
$$

Expressions (6.5) are verified solely by use of relations (6.9) and the cancellation of group elements and their inverses (when juxtaposed).

Assume further that

(ii) the commutator relations (6.9) continue to hold when $\boldsymbol{g}_{i m}$ is replaced by $\boldsymbol{\tau}\left(\boldsymbol{g}_{i m}\right)=A_{i}, i=1,2,3$

Then according to the substitution test once more, $\boldsymbol{\tau}:\left\{\boldsymbol{g}_{1 m}, \boldsymbol{g}_{2 m}, \boldsymbol{g}_{3 m}\right\} \rightarrow$ $\left\{A_{1}, A_{2}, A_{3}\right\}$ extends to a homomorphism $\boldsymbol{\tau}^{\prime}$ of $D_{m}$, which is surjective because $\boldsymbol{\tau}^{\prime}\left(D_{m}\right)$ includes a set of generators (of $\left.D_{m}\right)$. Note that, when (6.2) is used to express the commutator relations (6.7) in terms of $\boldsymbol{g}_{1 m}, \boldsymbol{g}_{2 m}, \boldsymbol{g}_{3 m}$, and $\boldsymbol{g}_{i m}$ is replaced by $A_{i}, i=1,2,3$ in (6.9), the effect is to replace $A_{i}$ in the commutator relations (6.7) by

$$
A_{1}^{a_{i 1}} \ldots A_{3}^{c_{i k}}, i=1,2,3 .
$$

Now note that $\phi^{\prime}$ and $\tau^{\prime}$ are mutually inverse homomorphisms, because $\phi^{\prime} \circ \tau^{\prime}$ represents the identity mapping on generators $\left\{\boldsymbol{g}_{1 m}, \boldsymbol{g}_{2 m}, \boldsymbol{g}_{3 m}\right\}$, and $\boldsymbol{\tau}^{\prime} \circ \boldsymbol{\phi}^{\prime}$ represents the identity on generators $\left\{A_{1}, A_{2}, A_{3}\right\}$. Hence $\boldsymbol{\phi}^{\prime}$ is an automorphism of $D_{m}$, and $\left(\phi^{\prime}\right)^{-1}=\boldsymbol{\tau}^{\prime}$. Recalling expression (6.10), note from (6.3) that

$$
\begin{aligned}
\phi^{\prime}\left(A_{1}^{a_{1 i}} \ldots A_{3}^{c_{k i}}\right) & =\left(\phi^{\prime}\left(A_{1}\right)\right)^{a_{i 1}} \ldots\left(\phi^{\prime}\left(A_{3}\right)\right)^{c_{k i}} \\
& =\left(\phi\left(A_{1}\right)\right)^{a_{i 1}} \ldots\left(\phi\left(A_{3}\right)\right)^{c_{k i}} \\
& =\boldsymbol{g}_{1 m}^{a_{i 1}} \ldots \boldsymbol{g}_{3 m}^{c_{k i}} \\
& =A_{i}, \quad i=1,2,3 .
\end{aligned}
$$

Recalling also the remark that precedes (6.10), we may rephrase assumption (ii) as

(iii) the commutator relations (6.7) continue to hold when $A_{i}$ is replaced by $\left(\phi^{\prime}\right)^{-1}\left(A_{i}\right), i=1,2,3$.

Hence we have shown the first part of the following lemma, and the second part is straightforward.

Lemma 2 Conditions (i) and (ii) above are sufficient that $\boldsymbol{\phi}$ and $\boldsymbol{\tau}$ extend to mutually inverse automorphisms of $D_{m}$. Conversely, if $\phi^{\prime}$ is an automorphism of $D_{m}$, and $\boldsymbol{\tau}^{\prime}$ is its inverse, then conditions (i), (ii) and (iii) hold.

We now use this lemma to compute the automorphisms of $D_{m} \subset S_{m}$, returning to the notation where the generators of $D_{m}$ are $A, B, C$.

Suppose that $\phi: D_{m} \rightarrow D_{m}$ is the change of generators defined by $\phi(A)=$ $\boldsymbol{g}_{1 m}, \boldsymbol{\phi}(B)=\boldsymbol{g}_{2 m}, \boldsymbol{\phi}(C)=\boldsymbol{g}_{3 m}$ where $\boldsymbol{g}_{1 m}, \boldsymbol{g}_{2 m}, \boldsymbol{g}_{3 m}$ are given by (5.4) and satisfy the conditions in section 5.2.2. Suppose also that $\boldsymbol{\tau}: D_{m} \rightarrow D_{m}$ is a change of generators such that $\boldsymbol{\tau}\left(\boldsymbol{g}_{1 m}\right)=A, \boldsymbol{\tau}\left(\boldsymbol{g}_{2 m}\right)=B, \boldsymbol{\tau}\left(\boldsymbol{g}_{3 m}\right)=C$ and let

$$
\boldsymbol{\tau}(A)=A^{p_{1}} B^{q_{1}} C^{r_{1}}, \quad \boldsymbol{\tau}(B)=A^{p_{2}} B^{q_{2}} C^{r_{2}}, \quad \boldsymbol{\tau}(C)=A^{p_{3}} B^{q_{3}} C^{r_{3}}
$$


for $p_{i}, q_{i}, r_{i} \in \mathbb{Z}, i=1,2,3$.

From the commutator relations (5.2) for $A, B$ and $C$ one can see from the Lemma 2 that if $\phi$ is to extend to an automorphism $\phi^{\prime}$ of $D_{m}$ then it must satisfy

$$
(\phi(A), \phi(B))=\phi(B)^{1-d} \phi(C)^{c} \quad \text { and } \quad(\phi(A), \phi(C))=\phi(B)^{b} \phi(C)^{1-a} .
$$

Since the commutators on the left-hand side of each expression above can be written as $B^{M} C^{N}$ for some $M, N \in \mathbb{Z}$, the power of $A$ in the expressions on the right-hand sides must be zero. This implies that

$$
\alpha_{2}(1-d)+\alpha_{3} c=0 \quad \text { and } \quad \alpha_{2} b+\alpha_{3}(1-a)=0 .
$$

Then $\alpha_{2}=\alpha_{3}=0$ since $a d-b c=1$ and $a+d \neq 2$. Similarly we deduce that $p_{2}=p_{3}=0$ if $\boldsymbol{\tau}$ extends to an automorphism $\boldsymbol{\tau}^{\prime}$ of $D_{m}$. Since $\boldsymbol{\tau}^{\prime} \circ \boldsymbol{\phi}^{\prime}$ is the identity, we must also have

$\boldsymbol{\tau}(\phi(A))=\boldsymbol{\tau}\left(A^{\alpha_{1}} B^{\beta_{1}} C^{\gamma_{1}}\right)=(\boldsymbol{\tau}(A))^{\alpha_{1}}(\boldsymbol{\tau}(B))^{\beta_{1}}(\boldsymbol{\tau}(C))^{\gamma_{1}}=A^{p_{1} \alpha_{1}} B^{M} C^{N}=A$

so $\alpha_{1} p_{1}=1$ and hence $\alpha_{1}=p_{1}=\zeta= \pm 1$. In addition we must have $\boldsymbol{\tau}(\phi(B))=B$ and $\boldsymbol{\tau}(\phi(C))=C$ from which we deduce that

$$
\left(\begin{array}{cc}
q_{2} & q_{3} \\
r_{2} & r_{3}
\end{array}\right)\left(\begin{array}{ll}
\beta_{2} & \beta_{3} \\
\gamma_{2} & \gamma_{3}
\end{array}\right)=\left(\begin{array}{ll}
1 & 0 \\
0 & 1
\end{array}\right) \quad \Rightarrow \quad \begin{gathered}
\beta_{2} \gamma_{3}-\gamma_{2} \beta_{3}:=\Delta= \pm 1 \\
q_{2} r_{3}-q_{3} r_{2}=\Delta
\end{gathered}
$$

Let us define

$$
\chi:=\left(\begin{array}{cc}
\beta_{2} & \beta_{3} \\
\gamma_{2} & \gamma_{3}
\end{array}\right) \in G L_{2}(\mathbb{Z}) .
$$

Assume now that $\alpha_{1}=\zeta$ and $\alpha_{2}=\alpha_{3}=0$. Then $\phi$ satisfies

$$
\begin{aligned}
& \phi(A)^{-1} \phi(B)^{-1} \phi(A) \phi(B)=\phi((A, B))=(\phi(A), \phi(B))=\phi(B)^{1-d} \phi(C)^{c} \\
& \phi(A)^{-1} \phi(C)^{-1} \phi(A) \phi(C)=\phi((A, C))=(\phi(A), \phi(C))=\phi(B)^{b} \phi(C)^{1-a} .
\end{aligned}
$$

Now

$$
\begin{aligned}
& \phi(A)^{-1} \phi(B)^{-1} \phi(A) \phi(B)=B^{x_{1}} C^{x_{2}} \\
& \phi(A)^{-1} \phi(C)^{-1} \phi(A) \phi(C)=B^{y_{1}} C^{y_{2}}
\end{aligned} \quad \text { where } \quad\left(\begin{array}{l}
x_{1} y_{1} \\
x_{2} y_{2}
\end{array}\right)=-\left(\theta^{-\zeta}-\mathbb{I}_{2}\right) \chi
$$

and

$$
\begin{aligned}
& \phi(B)^{1-d} \phi(C)^{c}=B^{x_{1}} C^{x_{2}} \\
& \phi(B)^{b} \phi(C)^{1-a}=B^{y_{1}} C^{y_{2}} \quad \text { where } \quad\left(\begin{array}{l}
x_{1} y_{1} \\
x_{2} y_{2}
\end{array}\right)=\chi\left(\mathbb{I}_{2}-\theta^{-1}\right) .
\end{aligned}
$$

So in order to determine the automorphisms of $D_{m}$ we therefore need to determine the matrices $\chi \in G L_{2}(\mathbb{Z})$ which satisfy

$$
\theta^{\zeta} \chi=\chi \theta \quad \text { for } \quad \zeta= \pm 1 .
$$

This condition is also sufficient that the conditions of Lemma 2 hold. 
6.2 Computing the automorphisms of $D$

Above, we showed that a change of generators $\phi$ extends to an automorphism of $D_{m}$ if it satisfies

$$
\phi(A)=A^{\zeta} B^{\beta_{1}} C^{\gamma_{1}}, \quad \phi(B)=B^{\beta_{2}} C^{\gamma_{2}}, \quad \phi(C)=B^{\beta_{3}} C^{\gamma_{3}},
$$

where $\zeta= \pm 1, \beta_{1}$ and $\gamma_{1}$ are arbitrary integers and the matrix of exponents $\beta_{i}, \gamma_{i} i=2,3$, denoted $\chi$ as in (6.13) has determinant \pm 1 and satisfies

$$
\theta^{\zeta} \chi=\chi \theta
$$

Here, following the work of Baake and Roberts [2], we summarise how to determine the matrices $\chi \in G L_{2}(\mathbb{Z})$ which satisfy $(6.14),(6.15)$ for a given matrix $\theta \in S L_{2}(\mathbb{Z})$.

As in [2], we define the set of symmetries of the matrix $\theta \in S L_{2}(\mathbb{Z})$ as

$$
S(\theta):=\left\{\chi \in G L_{2}(\mathbb{Z}): \theta \chi=\chi \theta\right\} .
$$

This is a subgroup of $G L_{2}(\mathbb{Z})$ and is the centralizer of $\theta$ in $G L_{2}(\mathbb{Z})$. If $\chi \in$ $G L_{2}(\mathbb{Z})$ satisfies $\chi \theta \chi^{-1}=\theta^{-1}$ then we say that $\chi$ is a reversing symmetry of $\theta$ and when such a $\chi$ exists we call $\theta$ reversible. We define the following subgroup of $G L_{2}(\mathbb{Z})$ as the reversing symmetry group of $\theta$,

$$
R(\theta):=\left\{\chi \in G L_{2}(\mathbb{Z}): \chi \theta \chi^{-1}=\theta^{ \pm 1}\right\} .
$$

It is a subgroup of the normalizer of the group generated by $\theta$ in $G L_{2}(\mathbb{Z})$ and clearly $S(\theta) \subset R(\theta)$. (If $H \subset G$, the normalizer of $H$ in $G$ is $\{a \in G: a H=H a\}$ ). Moreover, $S(\theta)$ is a normal subgroup of $R(\theta)$. There are two possibilities:

i) $R(\theta)=S(\theta)$. This occurs in cases where $\theta$ is not reversible, or if $\theta^{2}=\mathbb{I}_{2}$;

ii) The index of $S(\theta)$ in $R(\theta)$ is 2 so that $R(\theta)$ is a $C_{2}$-extension of $S(\theta)$ (where $C_{2}$ is the cyclic group of order 2 ).

Recall that if $\theta^{2}=\mathbb{I}_{2}$ then for $\theta$ to lie on a one parameter subgroup of $S L_{2}\left(\mathbb{R}^{3}\right)$, $\theta=-\mathbb{I}_{2}$ and this contradicts the fact that $\operatorname{tr}(\theta)>2$ in $S_{1}$. (We do not deal with the nilpotent case, where $\theta=\mathbb{I}_{2}$, either). So $R(\theta)=S(\theta)$ only when $\theta$ is not reversible. Given a matrix $\theta \in S L_{2}(\mathbb{Z})$, we want to compute $R(\theta)$ - there is a finite algorithm for this computation which we briefly summarize following Baake and Roberts [2]. First we consider $S(\theta)$.

\subsubsection{Computing $S(\theta)$}

Clearly $\theta^{m} \in S(\theta)$ for all $m \in \mathbb{Z}$. What other elements lie in $S(\theta)$, if any?

Since $\operatorname{tr}(\theta)>2$ the eigenvalues of $\theta, \lambda$ and $1 / \lambda$, are real, positive and distinct. One lies inside strictly inside the interval $[0,1]$ and the other strictly outside. Consequently $\theta$ can be diagonalised by a matrix $U$ which has entries in $\mathbb{Q}(\lambda)$, the smallest field extension of the rationals that contains $\lambda$, so that

$$
U^{-1} \theta U=\left(\begin{array}{ll}
\lambda & 0 \\
0 & \frac{1}{\lambda}
\end{array}\right) \quad \text { where } \theta=\left(\begin{array}{ll}
a & b \\
c & d
\end{array}\right), \quad U=\left(\begin{array}{cc}
b & b \\
\lambda-a & \frac{1}{\lambda}-a
\end{array}\right) .
$$


Suppose that $\chi \in S(\theta)$. Then $U^{-1} \chi U$ commutes with $U^{-1} \theta U$ and since only diagonal matrices can commute with a diagonal matrix with different diagonal entries,

$$
U^{-1} \chi U=\left(\begin{array}{cc}
\mu_{1} & 0 \\
0 & \mu_{2}
\end{array}\right), \quad \text { some } \mu_{1}, \mu_{2} \in \mathbb{Q}(\lambda) .
$$

Thus $S(\theta)$ contains all matrices $\chi \in G L_{2}(\mathbb{Z})$ which are diagonalised by $U$. Since $\chi \in G L_{2}(\mathbb{Z})$, it has eigenvalues $\mu_{1}, \mu_{2}$ which are algebraic integers. Also $\mu_{2}= \pm \mu_{1}^{-1}$ and hence $\mu_{1}$ and $\mu_{2}$ are units in $\mathcal{O}$, the maximal order of $\mathbb{Q}(\lambda)$ (i.e the intersection of $\mathbb{Q}(\lambda)$ with the set of algebraic integers).

A number theoretic argument, using Dirichlet's unit theorem [3], [5] shows that $\lambda= \pm u^{m}$ for some $u \in \mathbb{Q}(\lambda)$ which is also an algebraic integer, and some integer $m$, where $u$ is the so-called fundamental unit, in this context. (There is a finite algorithm for computing the fundamental unit which can be found in Borevich and Shafarevich [3]). The $m^{\text {th }}$ root of the matrix $\theta$ does not necessarily lie in $G L_{2}(\mathbb{Z})$, but there is a least integer $n$ such that

$$
M \equiv U\left(\begin{array}{ll}
u & 0 \\
0 & u^{-1}
\end{array}\right)^{n} U^{-1} \in G L_{2}(\mathbb{Z}),
$$

which may be determined when $u$ is known. Then $\theta=M^{p}$, where $p \equiv M / n \in$ $\mathbb{Z}$, and $M$ generates $S(\theta)$, modulo sign.

\subsubsection{Properties of $R(\theta)$}

We do not describe the computation of $R(\theta)$ in detail, since it is a complex procedure, but choose just to quote the following properties of the reversing symmetries in the particular case $\theta \in S L_{2}(\mathbb{Z})$.

First a matrix $\theta \in S L_{2}(\mathbb{Z})$ with $\operatorname{tr} \theta>2$ is reversible in $G L_{2}(\mathbb{Z})$ if and only if $\theta$ is conjugate in $G L_{2}(\mathbb{Z})$ to a matrix $k \in S L_{2}(\mathbb{Z})$ which has one of three forms: (i) $k$ is symmetric, (ii) $k$ is antisymmetric, (iii) the diagonal elements of $k$ are equal. There is an algorithm which decides whether or not two elements of $S L_{2}(\mathbb{Z})$ are conjugate in $G L_{2}(\mathbb{Z})$, see $[2]$.

The symmetries of $\theta$ (i.e. $S(\theta)$ ) can be found for each $\theta \in S L_{2}(\mathbb{Z})$. If $\theta$ has a reversing symmetry $\psi$, then all others reversing symmetries can be written in the form $\psi \nu, \nu \in S(\theta)$. All reversing symmetries have order 2 or 4 , and for each class of matrices $\theta \in S L_{2}(\mathbb{Z})$ (which have reversing symmetries) in turn, we have (i) $\psi$ is conjugate in $G L_{2}(\mathbb{Z})$ to $\left(\begin{array}{cc}0 & 1 \\ -1 & 0\end{array}\right),($ ii $) \psi$ is conjugate in $G L_{2}(\mathbb{Z})$ to $\left(\begin{array}{ll}0 & 1 \\ 1 & 0\end{array}\right),\left(\right.$ iii) $\psi$ is conjugate in $G L_{2}(\mathbb{Z})$ to $\left(\begin{array}{cc}1 & 0 \\ 0 & -1\end{array}\right)$.

\section{Extensions of automorphisms of $D$ to automorphisms of $S_{1}$}

A result of Gorbatsevich [11] states that if $S$ is a simply connected Lie group of type $(R)$ and $D$ is a discrete subgroup of $S$ such that $S / D$ is compact then 
every automorphism $\phi: D \rightarrow D$ can be extended uniquely to an automorphism $\widetilde{\phi}: S \rightarrow S$. A Lie group $S$ is of type $(R)$ if its Lie algebra $\mathfrak{s}$ is of type $(R)$ and a Lie algebra is of type $(R)$ if for each $\boldsymbol{x} \in \mathfrak{s}$ the eigenvalues of ad $\boldsymbol{x}$ are real. Here $\operatorname{ad} \boldsymbol{x}: \mathfrak{s} \rightarrow \mathfrak{s}$ is the adjoint action of $\boldsymbol{x}$ on $\mathfrak{s}$ and is defined as $\operatorname{ad} \boldsymbol{x}(\boldsymbol{y})=[\boldsymbol{x}, \boldsymbol{y}]$ for all $\boldsymbol{y} \in \mathfrak{s}$, where $[\cdot, \cdot]$ is the Lie bracket operation in $\mathfrak{s}$. One can compute that $S_{1}$ (and also $S_{m}$ ) is a Lie group of type $(R)$.

It follows that the automorphisms of $D$ are (restrictions of) elastic deformations of the continuum.

Here we show explicitly how the automorphisms of $D$ computed in section 6 extend uniquely to the automorphisms of $S_{1}$ computed in section 4 .

Recall that with respect to the basis $\left\{\boldsymbol{f}_{1}, \boldsymbol{f}_{2}, \boldsymbol{f}_{3}\right\}$ the Lie group automorphisms $\phi: S_{1} \rightarrow S_{1}$ are given by (4.24) and these automorphisms are uniquely determined by the values of $\epsilon \in\{0,1\}, \alpha, \beta, \gamma$ and $\delta \in \mathbb{R}$ in the corresponding Lie algebra automorphism $L$. Note that if we let

$$
\bar{M}=\left(\begin{array}{ll}
-b^{\prime}(0) & a^{\prime}(0)-k \\
-b^{\prime}(0) & a^{\prime}(0)+k
\end{array}\right),
$$

and recall the quantities defined in (4.23) then

$$
\begin{aligned}
& \phi\left(\boldsymbol{e}_{1}\right)=\phi\left(\left(\begin{array}{c}
\left.\bar{M}^{-T}\left(\begin{array}{l}
1 \\
0
\end{array}\right)\right) \\
0
\end{array}\right)=\left(\begin{array}{c}
\left.L^{\prime}(\epsilon) \bar{M}^{-T}\left(\begin{array}{l}
1 \\
0
\end{array}\right)\right), \\
0
\end{array}\right)\right. \\
& \phi\left(\boldsymbol{e}_{2}\right)=\phi\left(\left(\begin{array}{c}
\left.\bar{M}^{-T}\left(\begin{array}{l}
0 \\
1
\end{array}\right)\right) \\
0
\end{array}\right)\right)=\left(\begin{array}{c}
\left.L^{\prime}(\epsilon) \bar{M}^{-T}\left(\begin{array}{l}
0 \\
1
\end{array}\right)\right), \\
0
\end{array}\right) \\
& \phi\left(\boldsymbol{e}_{3}\right)=\phi\left(\boldsymbol{f}_{3}\right)=\left(\begin{array}{c}
F(B \xi)\left(\begin{array}{l}
l_{1}(\epsilon) \\
l_{2}(\epsilon)
\end{array}\right) \\
\xi
\end{array}\right),
\end{aligned}
$$

where we give components with respect to $\boldsymbol{f}_{1}, \boldsymbol{f}_{2}, \boldsymbol{f}_{3}$, and $\xi=(-1)^{\varepsilon}$.

Let $\phi_{D}: D \rightarrow D$ be an automorphism of the discrete subgroup $D$. Then we find that

$$
\begin{aligned}
& \phi_{D}\left(\boldsymbol{e}_{1}\right)=r_{m}^{-1}\left(\phi_{D_{m}}(B)\right)=r_{m}^{-1}\left(B^{\beta_{2}} C^{\gamma_{2}}\right)=\beta_{2} \boldsymbol{e}_{1}+\gamma_{2} \boldsymbol{e}_{2}=\left(\begin{array}{c}
\left.\bar{M}^{-T}\left(\begin{array}{c}
\beta_{2} \\
\gamma_{2}
\end{array}\right)\right), \\
0
\end{array}\right), \\
& \phi_{D}\left(\boldsymbol{e}_{2}\right)=r_{m}^{-1}\left(\boldsymbol{\phi}_{D_{m}}(C)\right)=r_{m}^{-1}\left(B^{\beta_{3}} C^{\gamma_{3}}\right)=\beta_{3} \boldsymbol{e}_{1}+\gamma_{3} \boldsymbol{e}_{2}=\left(\begin{array}{c}
\bar{M}^{-T}\left(\begin{array}{l}
\beta_{3} \\
\gamma_{3}
\end{array}\right) \\
0
\end{array}\right), \\
& \phi_{D}\left(\boldsymbol{e}_{3}\right)=r_{m}^{-1}\left(\boldsymbol{\phi}_{D_{m}}(A)\right)=r_{m}^{-1}\left(A^{\zeta} B^{\beta_{1}} C^{\gamma_{1}}\right)=\left(\begin{array}{c}
\bar{M}^{-T} \theta^{\zeta}\left(\begin{array}{c}
\beta_{1} \\
\gamma_{1}
\end{array}\right) \\
\zeta
\end{array}\right), \quad(7.2)
\end{aligned}
$$

where again we give components with respect to $\boldsymbol{f}_{1}, \boldsymbol{f}_{2}, \boldsymbol{f}_{3}$. 
Comparing (7.1) and (7.2) it can be shown that $\phi_{D}$ extends to $\phi$ if and only if $\xi=(-1)^{\epsilon}=\zeta$ and, firstly, if $\epsilon=0$, then

$$
\begin{array}{cc}
\alpha=\frac{\beta_{3}\left(a-\mathrm{e}^{-k}\right)}{b}+\gamma_{3}, & \gamma=-\frac{\mathrm{e}^{k}}{2 b\left(\mathrm{e}^{k}-1\right)}\left(\left(a-\mathrm{e}^{-k}\right) \beta_{1}+b \gamma_{1}\right), \\
\beta=\frac{\beta_{3}\left(a-\mathrm{e}^{k}\right)}{b}+\gamma_{3}, & \delta=-\frac{\mathrm{e}^{-k}}{2 b\left(\mathrm{e}^{-k}-1\right)}\left(\left(a-\mathrm{e}^{k}\right) \beta_{1}+b \gamma_{1}\right) .
\end{array}
$$

Similarly, if $\epsilon=1$ then

$$
\begin{array}{ll}
\alpha=-\frac{\beta_{3}\left(a-\mathrm{e}^{k}\right)}{b}-\gamma_{3}, & \gamma=\frac{\mathrm{e}^{-k}}{2 b\left(\mathrm{e}^{-k}-1\right)}\left(\left(a-\mathrm{e}^{k}\right) \beta_{1}+b \gamma_{1}\right), \\
\beta=-\frac{\beta_{3}\left(a-\mathrm{e}^{-k}\right)}{b}+\gamma_{3}, & \delta=\frac{\mathrm{e}^{k}}{2 b\left(\mathrm{e}^{k}-1\right)}\left(\left(a-\mathrm{e}^{-k}\right) \beta_{1}+b \gamma_{1}\right) .
\end{array}
$$

One can confirm that the extension of $\phi_{D}$ to $\phi: S_{1} \rightarrow S_{1}$, with these values of $\epsilon, \alpha, \beta, \gamma$ and $\delta$, is unique since the correspondence between $(\alpha, \beta, \gamma, \delta)$ and $\left(\gamma_{1}, \gamma_{3}, \beta_{1}, \beta_{3}\right)$ is one to one. Thus we have shown directly that every automorphism $\phi_{D}: D \rightarrow D$ extends uniquely to an automorphism $\phi: S_{1} \rightarrow S_{1}$.

\section{Conclusion}

These rather technical calculations may be briefly summarized, to reinforce the motivation for the work. First note from (1.22) that if $\left\{\boldsymbol{\ell}_{a}\right\}=\left\{\boldsymbol{\ell}_{a}(\mathbf{0})\right\}$ and $S$ are known, then so are the structure constants $\left(C_{i j k}\right) \equiv C$. Thus one may express the energy density $w\left(\left\{\boldsymbol{\ell}_{a}\right\}, S\right)$ alternatively as

$$
w^{+}\left(\left\{\ell_{a}\right\}, C\right) \text {. }
$$

Given that we have focussed on a canonical Lie group (with structure constants $C)$, the symmetry condition (1.9) becomes

$$
w^{+}\left(\left\{\boldsymbol{\ell}_{a}\right\}, C\right)=w^{+}\left(\left\{\tilde{\ell_{a}}\right\}, C\right) .
$$

In (8.1), $\left\{\boldsymbol{\ell}_{a}\right\}$ and $\left\{\tilde{\boldsymbol{\ell}}_{a}\right\}$ are (modulo exponentiation) different sets of generators of the discrete group which have denoted by $D$. When the generators $\left\{\boldsymbol{\ell}_{a}\right\},\left\{\tilde{\ell}_{a}\right\}$ are such that condition (6.14) holds, the mapping that takes $\left\{\boldsymbol{\ell}_{a}\right\} \rightarrow\left\{\tilde{\boldsymbol{\ell}}_{a}\right\}$ extends to an elastic deformation which maps the corresponding uniform crystal state to itself. Condition (6.14) is to be solved for matrices $\chi \in G L_{2}(\mathbb{Z})$, given the matrix $\theta \in S L_{2}(\mathbb{Z})$ which is associated with the dislocation density tensor via (4.4), (4.6), (4.11), (4.18). According to section 6.2.1, the solutions of (6.14) are determined, in the simplest case, by finding an appropriate root of $\theta$ (in particular, the root is to lie in $G L_{2}(\mathbb{Z})$ ). So in loose terms the energy density has a 'periodicity' determined by a particular root of (a quantity associated with) the dislocation density. That is to say, there is a periodicity in the energy, and hence a weakness in the strength of the 
corresponding crystal, determined by the distribution of defects. Moreover, in canonical form the points of $D$ represent a perfect lattice, and the 'discrete' Burgers' vectors (i.e the commutators of the generators) are confined to a certain plane in the lattice.

It will be a central future task to understand properties of the changes of sets of generators that do not satisfy (6.14), i.e.the 'inelastic symmetries', to facilitate the consideration of corresponding mechanical (or thermomechanical) problems - this seems to be difficult, however.

Acknowledgements We acknowledge the support of the UK Engineering and Physical Sciences Research Council through grant EP/G047162/1.

\section{References}

1. Auslander, L., Green, L., Hahn, F.: Flows on homogeneous spaces. Ann. Math. Stud. 53, NJ Princeton University Press, Princeton (1963)

2. Baake, M., Roberts, J. A. G.: Reversing symmetry group of $G L(2, \mathbb{Z})$ and $P G L(2, \mathbb{Z})$ matrices with connections to cat maps and trace maps. J. Phys. A. Math. Gen. 30, 1549-73 (1997)

3. Borevich, Z. I., Shafarevich,I. R.: Number Theory translated by N. Greenleaf for Scripta Technica, Academic Press, New York, London (1966)

4. Cermelli P., Parry, G. P.: The structure of uniform discrete defective crystals. Contin. Mech. Thermodyn. 18, 47-61 (2006)

5. Cohn,H.: Advanced Number Theory. Dover, New York (1980)

6. Coxeter, H. S. M., Moser, W. O. J.: Generators and Relations for Discrete Groups. Ergebnisse der Mathematik 14, Springer-Verlag, New York, Berlin, Heidelberg (3rd Ed) (1972)

7. Davini, C., Parry, G. P.: On defect preserving deformations in crystals. Int. J. Plast. 5, 337-369 (1989)

8. Davini, C.: A proposal for a continuum theory of defective crystals. Arch. Rat. Mech. Anal. 96, 295-317 (1986)

9. Davini, C.: Some remarks on the continuum theory of defects in solids. Int. J. Solids and Struct. 38 (6-7), 1169-1182 (2001)

10. Elzanowski, M. and Parry, G. P.: Material symmetry in a theory of continuously defective crystals. J. Elast. 74, 215-237 (2004)

11. Gorbatsevich, V. V.: Lattices in solvable Lie groups and deformations of homogeneous spaces. Math. USSR - Sb 20, 249-266 (1973)

12. Ha, K. Y., Lee, J. B.: Left invariant metrics and curvatures on simply connected threedimensional Lie groups. Math. Nachr. 282 (6), 868-898 (2009)

13. Johnson, D. L.: Presentations of Groups. 2nd Ed. Lond. Maths. Soc. Stu. Texts 15 Cambridge University Press, Cambridge (1997)

14. Kamber, F. W., Tondeur, P. H.: Flat manifolds with parallel torsion. J. Differ. Geom. 2, 385-389 (1968)

15. Kurosh, A.G.: The Theory of Groups, 2. Translated from the Russian by K. A. Hirsch, Chelsea, New York (1956)

16. Lovelock, D., Rund, H.:Tensors, Differential forms and Variational Priciples. Dover Publications Inc., New York (1989)

17. Magnus, W., Karrass, A., Solitar, D.: Combinatorial Group Theory. Dover, New York (1976)

18. Mal'cev, A.: On a class of homogeneous spaces. Isvestiya Akad. Nauk SSSR Ser., Mat. 13, 9-32 (1949). Am. Math. Soc. Transl. 39 (1949)

19. Nicks, R., Parry, G. P.: On symmetries of crystals with defects related to a class of solvable groups $\left(S_{1}\right)$. Math. Mech. Solids, 17 (6) 631-651 (2011) 
20. Nicks, R., Parry, G. P.: On symmetries of crystals with defects related to a class of solvable groups $\left(S_{2}\right)$. Math. Methods in the App. Sci., 35 1741-1755 (2012)

21. Parry, G.P.: Group properties of defective crystal structures. Math. Mech. Solids 8, 515-537 (2003)

22. Parry, G.P.: Rotational symmetries of crystals with defects. J. Elast. 94, 147-166 (2009)

23. Parry, G.P.: Elastic symmetries of defective crystals. J. Elast., 101, 101-120 (2010)

24. Parry, G.P., Sigrist, R.: Reconciliation of local and global symmetries for a class of crystals with defects. J. Elast., 107, (1) 81-104 (2012)

25. Pontryagin, L. S.: Topological Groups (2nd Ed.) Gordon and Breach, New York, London, Paris (1955)

26. Truesdell, C., Noll, W.: The Non Linear Field Theories of Mechanics. Handbuch der Physik, Band II/3, Springer, Berlin (1965). 